ACCEPTED MANUSCRIPT

\title{
Dose quantification in carbon ion therapy using in-beam positron emission tomography
}

To cite this article before publication: Harley Rutherford et al 2020 Phys. Med. Biol. in press https://doi.org/10.1088/1361-6560/abaa23

\section{Manuscript version: Accepted Manuscript}

Accepted Manuscript is "the version of the article accepted for publication including all changes made as a result of the peer review process, and which may also include the addition to the article by IOP Publishing of a header, an article ID, a cover sheet and/or an 'Accepted

Manuscript' watermark, but excluding any other editing, typesetting or other changes made by IOP Publishing and/or its licensors"

This Accepted Manuscript is @ 2020 Institute of Physics and Engineering in Medicine.

During the embargo period (the 12 month period from the publication of the Version of Record of this article), the Accepted Manuscript is fully protected by copyright and cannot be reused or reposted elsewhere.

As the Version of Record of this article is going to be / has been published on a subscription basis, this Accepted Manuscript is available for reuse under a CC BY-NC-ND 3.0 licence after the 12 month embargo period.

After the embargo period, everyone is permitted to use copy and redistribute this article for non-commercial purposes only, provided that they adhere to all the terms of the licence https://creativecommons.org/licences/by-nc-nd/3.0

Although reasonable endeavours have been taken to obtain all necessary permissions from third parties to include their copyrighted content within this article, their full citation and copyright line may not be present in this Accepted Manuscript version. Before using any content from this article, please refer to the Version of Record on IOPscience once published for full citation and copyright details, as permissions will likely be required. All third party content is fully copyright protected, unless specifically stated otherwise in the figure caption in the Version of Record.

View the article online for updates and enhancements. 


\section{Dose quantification in carbon ion therapy using in-beam positron emission tomography}

Harley Rutherford ${ }^{1,2}$, Andrew Chacon ${ }^{1,2}$, Akram Mohammadi ${ }^{3}$, Sodai Takyu ${ }^{3}$, Hideaki Tashima ${ }^{3}$, Eiji Yoshida ${ }^{3}$, Fumihiko Nishikido ${ }^{3}$, Theresa Hofmann ${ }^{4,5}$, Marco Pinto ${ }^{4}$, Daniel R. Franklin ${ }^{6}$, Taiga Yamaya ${ }^{3}$, Katia Parodi ${ }^{4}$, Anatoly B. Rosenfeld ${ }^{1,7}$, Susanna Guatelli ${ }^{1,7}$ and Mitra Safavi-Naeini ${ }^{1,2,8}$

1 Centre for Medical Radiation Physics, University of Wollongong, Wollongong NSW 2522 Australia

2 ANSTO, NSW, Australia

3 National Institute of Radiological Sciences (NIRS), National Institutes for Quantum and Radiological Science and Technology, 4-9-1 Anagawa, Inage-ku, Chiba 263-8555, Japan

4 Department of Experimental Physics-Medical Physics, Ludwig-Maximilians-Universität München, 85748 Garching b. Munich, Germany

${ }^{5}$ Europäisches Cyberknife Zentrum München, 81377 München, Germany

6 School of Electrical and Data Engineering, University of Technology Sydney, Sydney, NSW, Australia

7 Illawarra Health and Medical Research Institute, University of Wollongong, Wollongong NSW 2522 Australia

8 Brain and Mind Centre, University of Sydney, Sydney, NSW, Australia 


\begin{abstract}
.
This work presents an iterative method for the estimation of the absolute dose distribution in patients undergoing carbon ion therapy, via analysis of the distribution of positron annihilations resulting from the decay of positron-emitting fragments created in the target volume. The proposed method relies on the decomposition of the total positron-annihilation distributions into profiles of the three principal positron-emitting fragment species $-{ }^{11} \mathrm{C},{ }^{10} \mathrm{C}$ and ${ }^{15} \mathrm{O}$. A library of basis functions is constructed by simulating a range of monoenergetic ${ }^{12} \mathrm{C}$ ion irradiations of a homogeneous polymethyl methacrylate phantom and measuring the resulting onedimensional positron-emitting fragment profiles and dose distributions. To estimate the dose delivered during an arbitrary polyenergetic irradiation, a linear combination of factors from the fragment profile library is iteratively fitted to the decomposed positron annihilation profile acquired during the irradiation, and the resulting weights combined with the corresponding monoenergetic dose profiles to estimate the total dose distribution. A total variation regularisation term is incorporated into the fitting process to suppress high-frequency noise. The method was evaluated with fourteen different polyenergetic ${ }^{12} \mathrm{C}$ dose profiles in a polymethyl methacrylate target: one which produces a flat biological dose, ten with randomised energy weighting factors, and three with distinct dose maxima or minima within the spread-out Bragg peak region. The proposed method is able to calculate the dose profile with mean relative errors of $0.8 \%, 1.0 \%$ and $1.6 \%$ from the ${ }^{11} \mathrm{C},{ }^{10} \mathrm{C},{ }^{15} \mathrm{O}$ fragment profiles, respectively, and estimate the position of the distal edge of the SOBP to within an average of $0.7 \mathrm{~mm}, 1.9 \mathrm{~mm}$ and $1.2 \mathrm{~mm}$ of its true location.
\end{abstract}

\title{
1. Introduction
}

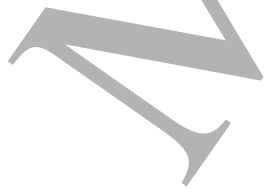

Carbon ion therapy is a form of radiotherapy in which accelerated carbon ions are used to deliver a therapeutic dose to the target volume $[1,2,3]$. This treatment modality offers several advantages over photon therapy, such as a well-defined energy-dependent depth of maximum dose shortly before the particles come to rest (known as the Bragg peak), and a high relative biological effectiveness (RBE), particularly at the distal end of the particle range $[2,4,5]$. The Bragg peak can be extended to deliver a uniform dose over a depth range by superimposing monoenergetic beams with different energies and fluences to form a polyenergetic beam, also known as a spread-out Bragg peak (SOBP) [1]. However, anatomical changes, errors in patient positioning and errors in the estimation of ion range may cause significant dose to be delivered outside the target region due to the steep dose gradients between the target region and surrounding healthy tissue [6].

During carbon ion therapy, a variety of target and projectile fragments are produced through nuclear inelastic collisions between ions in the beam and nuclei in the target volume in a process known as fragmentation $[4,5]$. Some of these fragments are radioactive and decay via positron emission after their creation, at a random time depending on their half-life. Positron emission tomography (PET) imaging of these decays can therefore be used for in-vivo treatment quality assurance, either after treatment (principally via longer-lived positron-emitting fragmentation products such 


\section{Dose quantification in carbon ion therapy using in-beam PET}

as ${ }^{11} \mathrm{C}$ or ${ }^{13} \mathrm{~N}$ ), or during treatment with in-beam PET (which primarily exploits the decay of short-lived fragmentation products such as ${ }^{10} \mathrm{C}$ and $\left.{ }^{15} \mathrm{O}\right)[7,8,9,10,11]$. Inbeam PET systems are one type of real-time treatment verification technique available for carbon ion therapy, and a number of in-beam scanner geometries have been proposed and are under development $[11,12,13,14,15,16,17,18]$.

The estimated distribution of positron annihilations obtained using a PET scanner during or after carbon ion therapy can be compared to the distribution predicted by the treatment planning system and/or a Monte Carlo simulation of the treatment $[19,20,21,22]$. The accuracy of treatment delivery then can be confirmed by evaluating the difference between the two distributions [23, 24]. However, accurately estimating the actual dose delivered from the spatial distribution of positron-emitting fragments is difficult due to the complex physics involved in charged particle energy deposition and nuclear inelastic collisions [5]. In addition, tissue-dependent metabolic washout of the produced activity can degrade the accuracy of these calculations [23, 25, 26].

One method for minimising the effect of metabolic washout on an acquired PET image is to apply a correction factor based on estimated washout rates of tissues in the treatment region [9]. Several studies have attempted to quantify the rate and impact of biological washout on distributions of positron annihilations obtained following the delivery of heavy ion beams, specifically in rat brains and rabbits, by fitting the observed PET signal in different tissues to a multi-exponential model with fast, medium and slow decay constants $[27,28,29,30,31,32]$. However, metabolic washout of positron-emitting isotopes created through fragmentation is a complex process and is dependent on many factors such as the specific treatment region, physiological state of the tumour, and the species of positron-emitting fragments present [29, 32, 33].

An alternative to correcting the impact of washout on a long-duration scan is to largely avoid washout in the first place by limiting the duration of the image acquisition - and acquiring the image during or immediately after irradiation - via the use of an in-beam or in-room PET scanner $[9,33,34]$. However, shorter scan times will result in a corresponding degradation in the signal to noise ratio in the resulting image.

In this work, a new method for PET image-based dose quantification in carbon ion therapy is proposed and evaluated using the Geant4 Monte Carlo simulation toolkit. A relationship between the observed positron annihilation and the delivered dose was developed based on the observation that the spatial distribution of each positron-emitting fragment species is unique for each monoenergetic carbon ion beam and target material. Our proposed technique estimates the delivered carbon ion dose by (1) measuring the distribution of the positron-emitting fragment yield within the target volume, (2) decomposing it into profiles of the individual positron-emitting fragment species (3) performing factor analysis on the decomposed activity profiles, using activity profiles resulting from the irradiation of a polymethyl methacrylate (PMMA) phantom with monoenergetic carbon ions as basis functions, and hence to calculate the proportional contribution of each energy to the beam. This approach can be tuned to minimise the effects of biological washout in tissues where this is likely to 
degrade the accuracy of the dose estimate.

This paper is divided into six sections, beginning with a summary of related work in Section 2. Section 3.1 describes the method used to generate a library of estimated positron-emitting fragmentation product distributions from simulated dynamic PET images of a PMMA phantom irradiated by a range of monoenergetic ${ }^{12} \mathrm{C}$ beams at different energies obtained using a Geant4 Monte Carlo simulation [35]. Section 3.2 details the procedure for estimating the weighting factors of energy components in a delivered SOBP ${ }^{12} \mathrm{C}$ beam from a dynamic PET image, and subsequently estimating the deposited dose profile. Results of the dose calculation procedure are presented in Section 4, and the effectiveness and accuracy of the proposed method is discussed in Section 5. Conclusions of this work are presented in Section 6.

\section{Related Work}

This section summarises different approaches proposed for PET-based treatment verification. These can be divided into indirect methods, based on comparing posttreatment PET images with predictions of Monte Carlo simulations or analytic models, and direct methods which estimate the dose distribution corresponding to the observed PET image. A number of techniques utilising both approaches have been developed for both proton and carbon therapy.

\subsection{Indirect PET-based dose verification}

Enghardt et al. developed one of the first methods for verifying a delivered dose distribution in carbon therapy by comparing a PET signal acquired following the treatment with an expected positron annihilation distribution [23]. This method produced an expected positron annihilation distribution using Monte Carlo simulations and compared it to the experimental PET signal to detect positioning errors and variations in local tissue density, before quantifying the difference between the recalculated and planned dose using the treatment planning system [23, 36]. Parodi and Bortfeld then proceeded to develop a method to estimate the expected PET signal from an intended dose map in proton therapy through convolution of the planned dose distribution with an analytical Gaussian-powerlaw filter function [37]. Parodi et al. and Attanasi et al. later applied this approach to clinical data, and Frey et al. integrated the method into a full treatment planning system (TPS) [9, 24, 38, 39]. These studies demonstrate the potential for indirect dose verification in charged particle therapy through comparison of the theoretically-predicted positron annihilation distribution and the experimentally acquired positron activity distributions, and the approach has been used in a number of subsequent investigations [19, 20, 21, 40, 41, 42]. However, a direct quantitative comparison between planned and delivered doses rather than positron annihilation distributions is preferable, as the dose is the quantity of interest [43]. 
Dose quantification in carbon ion therapy using in-beam PET

carbon ion therapy, and developed an analytical model to predict the positron-emitting fragment distribution from the depth-dose distribution [37, 44]. The filtering approach was used to predict the distal edge of the fragment distribution, and an analytical model fitted to data generated from Geant4 Monte Carlo simulations was used to estimate the shape of the distribution. The approach was validated against positronemitting fragment distributions obtained from Monte Carlo simulations, and was found to estimate the position of the distal edge of the Bragg peak to within $0.6 \mathrm{~mm}$ for homogeneous and $1.3 \mathrm{~mm}$ for inhomogeneous phantoms, with mean relative errors to the maximum of the distributions below $4 \%$ [44].

\subsection{Direct approaches to dose estimation}

Remmele et al. proposed a deconvolution-based approach to the inverse dose estimation problem based on the filter kernel developed by Parodi and Bortfeld, combined with either total variation (TV) or Tikhonov-Miller (TM) regularisation to enforce smoothness in the dose distribution [45]. Remmele et al. demonstrated that their method could recover accurate dose distributions in proton therapy from PET signals obtained from Monte Carlo simulations, and also PET signals experimentally obtained from both a PMMA phantom and a head and neck patient following a 2 Gy irradiation. While excellent results were obtained for the simulation data, artefacts in the experimental PET images resulted in significant errors in the estimation of the position of the distal edge of the spread-out Bragg peak, averaging 2-3 $\mathrm{mm}$ and with a maximum of $8 \mathrm{~mm}$. Aiello et al. propose a similar approach (also using Tikhonov regularisation) to the inverse dose estimation problem in proton therapy [43]. The proposed technique was evaluated in simulation using both a pristine proton Bragg peak and SOBP, simulated in homogeneous and heterogeneous phantoms using the FLUKA Monte Carlo simulation package [46]. The authors state that good agreement between the simulated ground truth and estimated dose distributions was obtained in the distal fall-off region of the pristine Bragg peak and SOBP. However, significant discrepancies were observed in the proximal regions of both the pristine Bragg peak and SOBP in the heterogeneous phantom [43].

Fourkal et al. also developed a deconvolution-based approach for direct dose verification in proton therapy, with the filter function analytically calculated using the $\beta^{+}$activation cross-section and proton energy fluence spectrum [47, 48]. The method was implemented for a parallel-opposed proton beam arrangement and simulated using FLUKA [46]; the dose distribution was estimated from the PET image to within $2 \%$ of the ground truth dose delivered in a homogeneous tissue phantom. Extending this method for heterogeneous targets such that it can be used for in-vivo dose reconstruction for patients would require substantial further development of the optimisation technique; the method would also need to be substantially modified for it to be applicable to carbon or other heavy ions. To our knowledge, no further work on this technique has been published as of 2020 [48]. 
Hofmann et al. recently reported the first deconvolution-based approach for estimating the dose distribution in carbon ion therapy [49]. Construction of the filter function used in this method uses a generalisation of the approach originally proposed by Parodi and Bortfeld in 2006 [37]. The filter kernel was iteratively optimised to minimise the error between the observed positron-emitting fragment distribution and the convolution of the kernel with the depth dose distribution obtained from Monte Carlo simulations [37]. Monte Carlo simulation was used to deliver monoenergetic ${ }^{12} \mathrm{C}$ ion beams to homogeneous and heterogeneous targets, and depth dose distributions were estimated based on the simulated fragment distributions to within a mean relative absolute error (relative to the maximum value) of $1 \%$ in homogeneous media, and $2 \%$ in heterogeneous media. Polyenergetic ${ }^{12} \mathrm{C}$ beams were delivered to homogeneous and heterogeneous phantoms in simulation, and the deconvolution approach estimated the depth dose distribution with an average mean relative absolute error of $2 \%$. The deconvolution approach was applied to measured fragment distributions in homogeneous and heterogeneous targets, and estimated the dose with a mean relative absolute error of $10 \%$ compared to the predictions of Monte Carlo simulations. These deviations were mainly attributed to the discrepancy between Monte Carlo simulated and measured fragment distributions. Finally, the deconvolution approach estimated the dose delivered by a simulated monoenergetic ${ }^{12} \mathrm{C}$ beam in a CT-derived model of a patient's head, based on the simulated fragment distribution, with a mean relative absolute error of $0.24 \%$ compared to the Monte Carlo simulation. This study demonstrates the feasibility of deconvolved PET image-based dose estimation methods to carbon ion therapy; however, at this stage, the effects of biological washout are not considered.

Inaniwa et al. developed a maximum likelihood expectation maximisation (MLEM) algorithm to predict the depths of the proximal and distal edges of SOBP ion beams, and later extended this method to estimate the shape of the deposited dose distribution $[50,51]$. Most recently, Masuda et al. proposed a technique for estimating the dose distribution in proton therapy which combines the filtering approach with a MLEM algorithm [52]. The MLEM algorithm was implemented to iteratively estimate the dose delivered by monoenergetic and SOBP proton beams in water, based on distributions of positron-emitting fragments acquired using an in-beam planar PET scanner. A MLEM algorithm was also used in/conjunction with the Monte Carlo-based software PHITS to iteratively calculate the filter functions, which were used in the dose estimation, and in the calculation of expected PET signals from intended dose maps [53]. Dose distributions were estimated in 1D and 2D using this method, with an accuracy of $7 \%$ for monoenergetic proton beam deliveries and $12 \%$ for SOBP proton beam deliveries. The authors claim the approach has advantages over the deconvolution-based algorithm used by Hofmann et al. particularly with regards to the speed of the dose calculation. However, the technique has yet to be adapted to carbon and other heavy ion therapies, and similarly to Hofmann et al. does not consider the effect of biological washout on the dose estimation.

In summary, several promising deconvolution-based solutions have been proposed 
Dose quantification in carbon ion therapy using in-beam PET

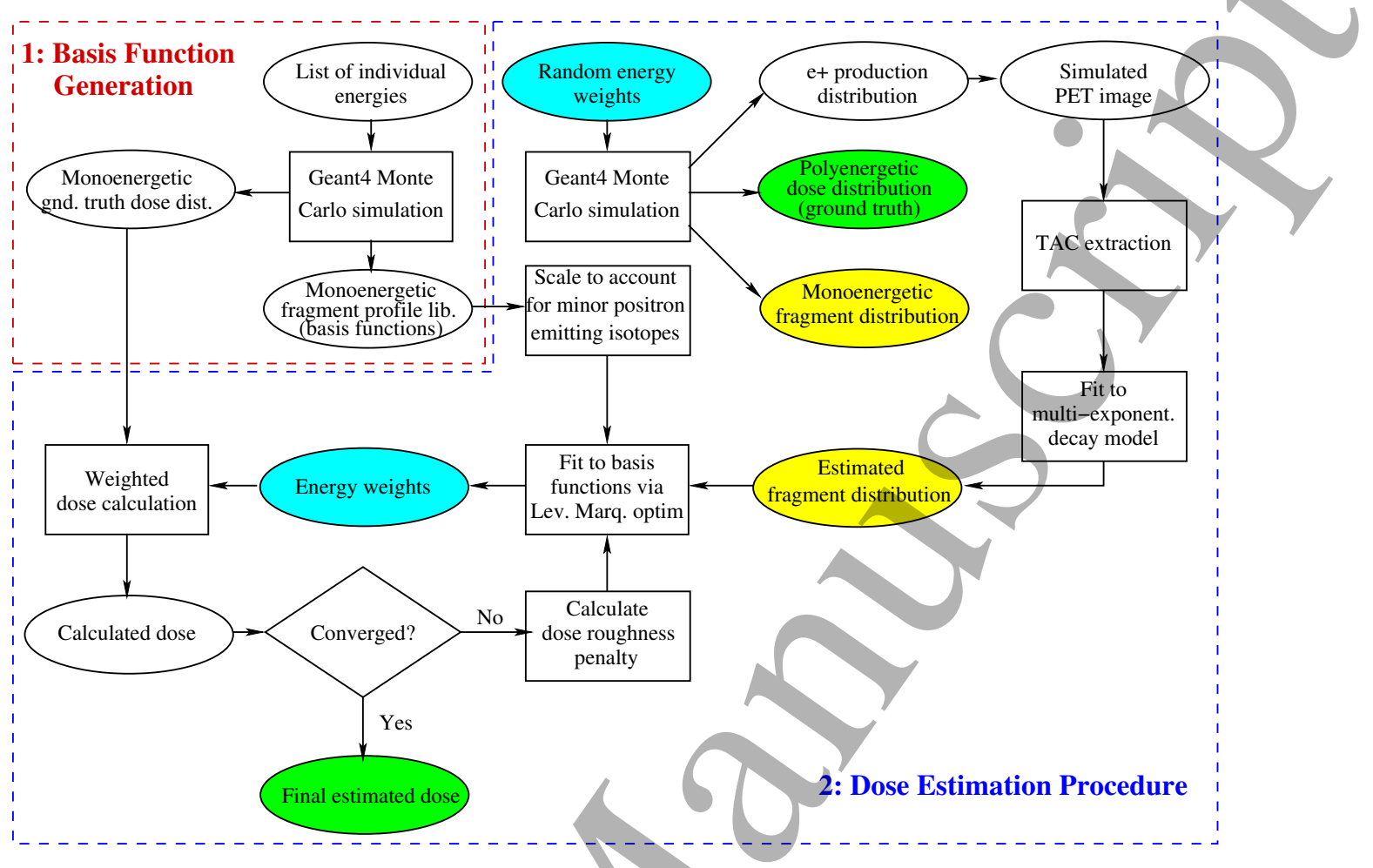

Figure 1. Flowchart summarising the method for dose calculation from a positron activity distribution induced by carbon ion irradiation. The red box illustrates the process of basis function generation (Section 3.1). The process of dose estimation is shown in the blue box (Section 3.2).

for the problem of estimating the dose distribution from the observed positron annihilation distribution resulting from the irradiation of a target by proton and heavy ion beams. However, the effects of biological washout have not been considered as yet. The approach proposed in this paper instead estimates the dose deposited by ${ }^{12} \mathrm{C}$ SOBPs from the observed positron-emitting fragment distribution obtained via a decomposition of the PET signal into spatial distributions of individual positron-emitting fragment species rather than the total activity map, which will allow the effects of biological washout to be minimised by selecting specific positron-emitting isotopes with short halflives. This will enable a reduction in the PET acquisition time according to the half-life of the selected positron-emitting isotope, and consequently the activity distribution will be degraded by washout to a lesser extent during the imaging process. The proposed method is validated for three positron-emitting fragment species with different half-lives.

\section{Materials and Methods}

In this section, the proposed method for calculating the dose distribution delivered by a polyenergetic ${ }^{12} \mathrm{C}$ irradiation based on the observed distribution of positron-emitting fragments is presented. A detailed overview of the method is shown in Figure 1. In summary, a dynamic PET image of the positron annihilations within the target resulting 
from irradiation by a polyenergetic carbon ion beam is decomposed along the beam path into the initial $(t=0)$ spatial profiles of each of the three principal positron-emitting radioisotope components present $-{ }^{11} \mathrm{C},{ }^{10} \mathrm{C}$ and ${ }^{15} \mathrm{O}$. Since this model neglects the remaining minor positron-emitting fragments, the profiles overestimate the-numbers of the three principal positron-emitting radionuclides; therefore, an empirically-derived scale factor is applied to compensate, resulting in an unbiased estimate of each of the true positron-emitting fragment profiles. A reference library of the ground-truth distributions of ${ }^{11} \mathrm{C},{ }^{10} \mathrm{C}$ and ${ }^{15}$ (basis functions) resulting from the irradiation of the PMMA target with individual monoenergetic carbon ion beams is generated using a combination of Monte Carlo simulations and interpolation; corresponding ground-truth dose profiles for each energy are also recorded. A linear combination of these fragment profiles is then fitted to the observed fragment distribution separately for each positronemitting fragment species, resulting in three estimates of the contributions of each energy component to the original polyenergetic beam. A regularisation term is included in the cost function while iteratively fitting the observed fragment distribution to suppress high-frequency noise in the estimated dose profile. Finally, the estimated weights are finally used to produce a composite estimate of the corresponding dose distribution along the beam path.

The method used to generate the positron-emitting fragment and dose profiles for the monoenergetic ${ }^{12} \mathrm{C}$ beams is described in detail in Section 3.1. All Monte Carlo simulations were performed with the Geant4 toolkit (version 10.2.p03) [35, 54]. The Geant4 QGSP_BIC_HP physics list was chosen to model hadronic processes, and electromagnetic processes were modeled using G4EmStandardPhysics_option3, based on the recommendation of a comparative study of Geant4 hadronic ion inelastic physics models by Chacon et al. [55]. The dose distribution estimation procedure is explained in detail in Section 3.2. The performance of the proposed method is evaluated using simulations of synthetic random SOBPs, as described in Section 3.3.

\subsection{Basis Function Generation}

Simulated monoenergetic ${ }^{12} \mathrm{C}$ beams with energies of 200, 215, 230, 245, 260, 275, $290,305,320$ and $350 \mathrm{MeV} / \mathrm{u}$ and a total particle fluence of $1.0 \times 10^{8}$ particles were delivered to a PMMA target phantom with dimensions of $100 \times 100 \times 300 \mathrm{~mm}^{3}$ using the Geant4 toolkit. The beam was modeled with a circular Gaussian intensity profile with a diameter of $8 \mathrm{~mm}$ full width at half maximum (FWHM) and an energy spread of $\sigma=0.2 \%$ (chosen based on previously published values for a ${ }^{12} \mathrm{C}$ ion pencil beam $[55,56])$. The ${ }^{12} \mathrm{C}$ ion beams were delivered to the phantom across a simulated air gap of $175 \mathrm{~cm}$ from the beam origin to the surface of the phantom, and a scoring resolution of $1.5 \mathrm{~mm}^{3}$ (based on current in-beam PET scanner attributes [56, 57]) was used to record the spatio-temporal distribution of positron-annihilation positions. Data were acquired for 36 minutes following beam delivery and binned into 5 second temporal frames. Additionally, the deposited dose was recorded with a spatial resolution of $1 \mathrm{~mm}^{3}$, 
normalised per incident particle.

Profiles of positron-emitting fragments, with activity normalised per primary particle, were constructed by selecting a $2 \mathrm{D}$ region of interest (ROI) in the transverse plane, with a width equal to the full width at tenth maximum (FWTM) of the beam for each voxel along the path of the beam, and scoring the annihilation positions of positrons originating from the decay of ${ }^{11} \mathrm{C},{ }^{10} \mathrm{C}$ and ${ }^{15} \mathrm{O}$ fragments in each slice. Deposited onedimensional profiles of energy deposition per primary particle were also constructed by summing the deposited energy scored in a FWTM ROI in the transverse plane around each voxel along the path of the beam. An initial library of one-dimensional positronemitting fragment profiles and deposited dose profiles was construeted by repeating this procedure for the 10 simulated monoenergetic ${ }^{12} \mathrm{C}$ ion beams delivered to the PMMA phantom. PMMA was chosen as the target material as it contains both oxygen and carbon, and yields a range of fragmentation products similar to that produced by heavy ion irradiation of human adipose tissue. In addition, PMMA and human adipose tissue have a similar electron density, resulting in a similar depth-dose profile [58].

To reduce the computational workload required to construct a complete library of dose and activity profiles, an interpolation procedure was used to calculate monoenergetic dose profiles and corresponding ${ }^{11} \mathrm{C},{ }^{10} \mathrm{C}$ and ${ }^{15} \mathrm{O}$ positron annihilation profiles for the beam energies between the specific values directly simulated in Geant4 $[59,60]$. Critical parameters of the simulated deposited dose and fragment profiles - such as depth and magnitude of the Bragg peaks - are extracted and linearly interpolated to estimate the values for the intermediate monoenergetic beams. For each new energy, the fragment profiles from the nearest two simulated beam energies are stretched or compressed to match the estimated Bragg peak magnitude and depth. The estimated positron-emitting fragment profile of the target incident beam energy is the average of these two estimates. The energies of simulated monoenergetic ${ }^{12} \mathrm{C}$ beams are separated by a maximum of $30 \mathrm{MeV} / \mathrm{u} \ddagger$.

At the conclusion of this step, a set of spatial profiles of the quantity of ${ }^{11} \mathrm{C},{ }^{10} \mathrm{C}$ and ${ }^{15} \mathrm{O}$ fragments produced during irradiation, and the corresponding dose profiles is created for monoenergetic beams with energies from $200 \mathrm{MeV} / \mathrm{u}$ to $350 \mathrm{MeV} / \mathrm{u}$ in steps of $1 \mathrm{MeV} / \mathrm{u}$.

\subsection{Dose Quantification Method}

The dose quantification procedure is summarised in Figure 1. A cumulative activity profile, $A_{S O B P, k}$, is generated by the delivery of a $\mathrm{SOBP}{ }^{12} \mathrm{C}$ beam according to $(1)$ :

$$
A_{S O B P, k}=\sum_{i} W_{i} \sum_{j} N_{i, j}(k) \times e^{\frac{-t \ln 2}{\tau_{j}}}
$$

A greater energy separation reduces the accuracy of this method, as range straggling changes the peak-to-entrance ratio of fragment yield profiles at a non-linear rate, which this approach would not accurately model. The interpolation method is validated in Appendix A. 


\section{Dose quantification in carbon ion therapy using in-beam PET}

$A_{S O B P, k}$ is a weighted summation of positron activity maps produced by each monoenergetic beam, weighted by a factor $W_{i}$, at a time $t$ after injection of the primary particles (assuming simultaneous injection of all particles). The $i$ th monoenergetic beam produces a number of positron-emitting fragments, $N_{i, j}(k)$, of species $j$ with half-life $\tau_{j}$, in the $k$ th voxel, normalised per incident particle.

One-dimensional estimates of the spatial profiles of ${ }^{11} \mathrm{C},{ }^{10} \mathrm{C}$ and ${ }^{15} \mathrm{O}$ fragment production in the image are generated by extracting a time activity curve (TAC) from a 2D ROI normal to the beam around each voxel along the path of the beam, defined by the FWTM of the beam in the transverse plane. Each extracted TAC is parametrically fitted with a multi-exponential model equation using the Levenburg-Marquardt (LM) error minimisation algorithm to obtain an estimate of the initial activity $A_{0}$ of each positron-emitting fragment species in each voxel $[61,62,63]$. Total activity as a function of time $t$ in a volume with initial activities $A_{0,11} \mathrm{C}, A_{0,{ }^{10} \mathrm{C}}$ and $A_{0,{ }^{15} \mathrm{O}}$ of ${ }^{11} \mathrm{C},{ }^{10} \mathrm{C}$ and ${ }^{15} \mathrm{O}$ respectively, is given by (2):

$$
A_{\text {total }}(t)=A_{0,{ }_{11} C} e^{-\ln t / \tau_{11} C}+A_{0,{ }^{10} C} e^{-\ln t / \tau_{10} C}+A_{0,{ }^{15} O} e^{-\ln t / \tau_{15} O}
$$

where $\tau_{11} \mathrm{C}, \tau_{{ }^{10} \mathrm{C}}$ and $\tau_{15} \mathrm{O}$ are the half-lives of ${ }^{11} \mathrm{C}(20.39 \mathrm{~min}),{ }^{10} \mathrm{C}(19.26 \mathrm{sec})$ and ${ }^{15} \mathrm{O}(122.24 \mathrm{sec})$ respectively.

The substantially longer half-life of ${ }^{11} \mathrm{C}$ relative to ${ }^{10} \mathrm{C}$ and ${ }^{15} \mathrm{O}$ results in the number of ${ }^{10} \mathrm{C}$ and ${ }^{15} \mathrm{O}$ nuclei decaying to anegligible quantity by 20 minutes post beam delivery; therefore, the final 15 minutes of each TAC can be solely attributed to the ${ }^{11} \mathrm{C}$ fragment component [64]. This information was used to reduce the dimensionality of the problem of finding the relative contributions of the shorter-lived isotopes, ${ }^{10} \mathrm{C}$ and ${ }^{15} \mathrm{O}$, in the earlier part of the time-activity curve.

Equation (2) implicitly assumes that all positron annihilations have resulted from the decay of ${ }^{11} \mathrm{C},{ }^{10} \mathrm{C}$ and ${ }^{15} \mathrm{O}$ nùclei. However, since several additional miscellaneous positron-emitting fragmentation products are also present, the number of ${ }^{11} \mathrm{C},{ }^{10} \mathrm{C}$ and ${ }^{15} \mathrm{O}$ nuclei tends to be-overestimated $\left({ }^{10} \mathrm{C}\right.$ in particular). To correct this overestimation, an empirical multiplicative scaling factor for each of the three estimated fragment profiles is calculated for all of the simulated monoenergetic ${ }^{12} \mathrm{C}$ beams such that the mean squared error between the estimated and ground truth fragment profiles is minimised, thereby compensating for the absence of the miscellaneous radionuclides. Across all of the simulated energies and fragment profiles, the standard deviation of the scaling factors was consistently low - between $2.2 \%$ and $3.6 \%$ of the mean. Therefore, by applying the mean scaling factor to the (over)estimated fragment profile, an unbiased estimate of the true fragment distribution can be reliably recovered.

To estimate the dose distribution, a linear combination of the one-dimensional monoenergetic fragment distributions (see Section 3.1) is iteratively fitted to the SOBP region of observed spatial fragment profiles to estimate the relative contributions of each energy component. However, the problem is ill-posed, and the linear combination which minimises the mean squared error between the observed and fitted fragment distributions can result in physically infeasible high-frequency fluctuations in the estimated dose 


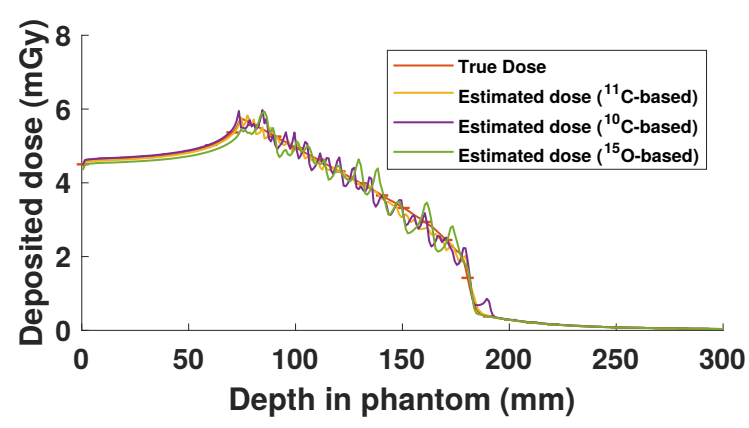

(a) Not regularised

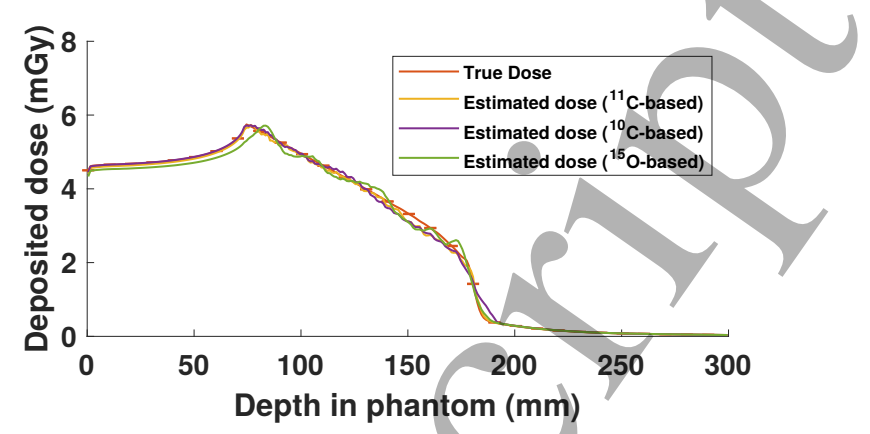

(b) Regularised

Figure 2. Results of the dose estimation procedure, with no regularisation (2(a)) and with total variation (L1) regularisation applied $(2(\mathrm{~b}))$, for one ${ }^{12} \mathrm{C}$ SOBP.

distribution. Therefore, a total variation (L1) regularisation term is included in the cost function to suppress these high-frequency fluctuations (since the dose distribution is assumed to be smooth and continuous between the proximal and distal edges of the SOBP). The problem may then be expressed as finding the optimal weight vector $\mathbf{W}_{\text {opt }}$ such that:

$$
\mathbf{W}_{\text {opt }}=\underset{\mathbf{W}}{\operatorname{argmin}}\left\{\left\|\mathbf{N}_{j} \mathbf{W}-\mathbf{A}_{\text {true }, j}\right\|^{2}+\alpha_{j} R_{f i t, j}(\mathbf{W})\right\}
$$

The first part of the cost function to be minimised is the sum of the squared errors between the ground truth fragment distribution $A_{\text {true }, j}$ and fitted fragment distribution $\mathbf{N}_{j} \mathbf{W}$ in the SOBP region, where $\mathbf{N}_{j}$ is the matrix of monoenergetic fragment production profiles corresponding to fragment species $j$, with columns representing increasing energy and rows representing depth along the path of the beam. The second part is a total variation (L1) regularisation term $R_{f i t, j}$, moderated by a factor $\alpha_{j}$ to adjust the degree of high-frequency suppression. $R_{f i t, j}$ is determined by calculating the dose distribution from the fragment weights in each iteration, transforming the profile into the frequency domain via a discrete cosine transform, and calculating the sum of absolute energy components above a critical threshold frequency $f_{t}$. This frequency is determined by computing the DCT of the planned dose distribution, setting frequency components above $f_{t}$ to zero, performing an inverse $\mathrm{DCT}$ and calculating the root mean squared error between the result and the original planned dose distribution; $f_{t}$ is progressively increased until the error is less than $0.25 \%$. The presence of any spectral components above $f_{t}$ in the estimated dose profile is therefore penalised. The highest frequency components in the planned dose distribution result from discontinuities in the dose distribution - principally, the proximal and distal edges of the SOBP. The optimal value of $\alpha_{j}$ is also iteratively calculated for each polyenergetic beam, and the average optimal value calculated for subsequent fitting of spatial fragment distributions. The effect of the regularisation term (after application of the target material-dependent correction factor) on the dose estimates for one ${ }^{12} \mathrm{C}$ SOBP is illustrated in Figure 2.

The fitting procedure is performed separately for each of the spatial ${ }^{11} \mathrm{C},{ }^{10} \mathrm{C}$ and ${ }^{15} \mathrm{O}$ 


\section{Dose quantification in carbon ion therapy using in-beam PET}

profiles, resulting in three sets of estimated weights of the different energy components of the original particle beam. While biological washout is not modeled in this work, we hypothesise that the most appropriate estimate can be selected depending on the irradiated tissue, its vascularisation, and on the most prominent processes of biological washout. Each set of weights provides an estimate of the number of incident particles of each energy originally used to generate the SOBP. Finally, the dose distributions from the individual monoenergetic components $E_{i, k}$ are summed using the weighting factors $W_{i}$ derived in the previous step, according to (4), to derive the estimate of the total deposited dose:

$$
E_{S O B P, k}=\sum_{i} W_{i} \times E_{i, k}
$$

\subsection{Performance Evaluation}

The performance of the proposed method was evaluated on Monte Carlo simulated ${ }^{12} \mathrm{C}$ ion beams with a variety of random dose distributions. The dose profiles estimated using the distribution of fragment species $j$ were compared with the ground truth values using the mean relative error (MRE), defined relative to the maximum of the ground truth dose distribution in the region $r$ :

$$
\operatorname{MRE} E_{r}\left(D_{\text {true }, j}(k), D_{e s t, j}(k)\right)=\frac{1}{n} \sum_{m=1}^{n} \frac{\left|D_{\text {true }, j}\left(k_{m}\right)-D_{e s t, j}\left(k_{m}\right)\right|}{\max _{r}\left(D_{\text {true }, j}\left(k_{r}\right)\right)}
$$

The MRE was evaluated in three regions: the entrance, SOBP and tail. The SOBP region is defined as the region between the most proximal and most distal Bragg peaks. The entrance region and tail region are then defined as the regions proximal and distal to the SOBP region, respectively. The MRE was also evaluated across the entire distribution for a direct comparison with the results of Hofmann et al. (2019a) [49].

Monte Carlo simulations, were performed for 14 different ${ }^{12} \mathrm{C}$ SOBPs, each with a total of $1.0 \times 10^{8}$ primary particles, in a PMMA target phantom. The SOBPs were composed of up to 76 monoenergetic beams with energies ranging from $200 \mathrm{MeV} / \mathrm{u}$ to $350 \mathrm{MeV} / \mathrm{u}$. The first profile used a set of weights designed to produce a flat biological dose; the next ten used randomised weight factors of between 1 and 10 for each energy; finally, the last three included well-defined dose maxima and/or minima within the spread-out Bragg peak region (Figure 3). Random variation was included in the weights to provide a more robust evaluation of the method. The statistical error in fragment yield and deposited dose profiles was calculated by dividing the delivered monoenergetic ${ }^{12} \mathrm{C}$ beams into 20 populations of $5.0 \times 10^{6}$ particles, and calculating the standard deviation in each voxel. Spatial fragment profiles are generated by fitting the decay model (2) to the simulated PET image; the energy weight estimation procedure is then applied to the profiles obtained from each simulation and composite dose estimate profiles generated; these are compared to the ground truth profiles, with the MRE evaluated across the entire distribution, and in the entrance, SOBP and tail regions. 

438

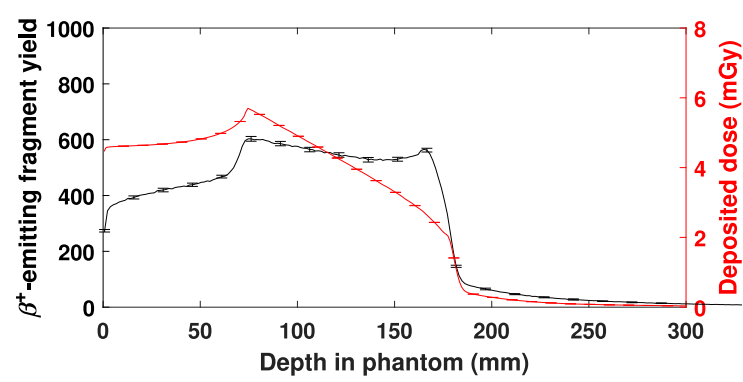

(a) Flat beam

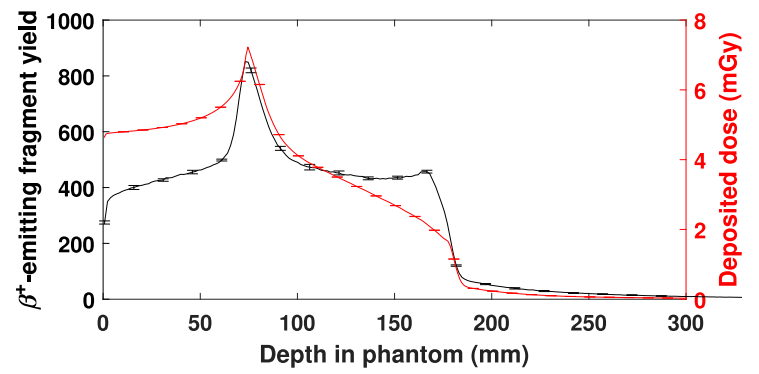

(c) Beam 12 (proximal maximum)

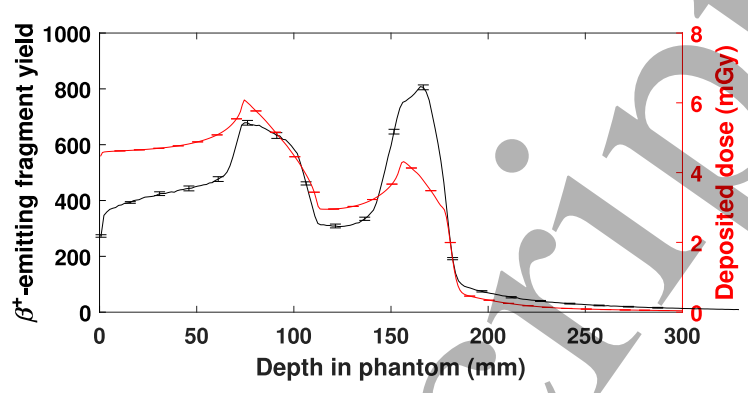

(b) Beam 11 (proximal and distal maxima)

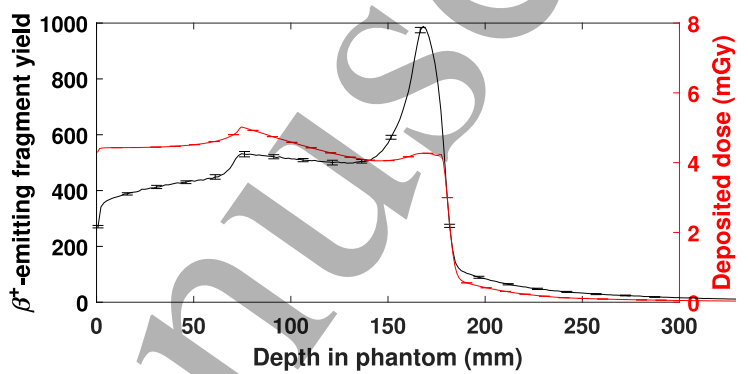

(d) Beam 13 (distal maximum)

Figure 3. Total positron-emitting fragment profiles (black) and deposited dose profiles (red) resulting from one simulated polyenergetic ${ }^{12} \mathrm{C}$ beam designed to produce a flat biological dose (a), and three simulated polyenergetic ${ }^{12} \mathrm{C}$ beams with distinct dose maxima or minima within the spread-out Bragg peak region (b)-(d).

The position of the distal edge of the SOBP was located (both for the ground truth and estimated dose profiles) by first differentiating the profile, and then identifying the deepest point at which the absolute gradient exceeds $0.03 \mathrm{~Gy} / \mathrm{m}$ (indicating the onset of the SOBP).

\section{Results}

The dose calculation procedure was implemented for the 14 different ${ }^{12} \mathrm{C}$ SOBP beams, each composed of a linear combination of monoenergetic components with energies from $200 \mathrm{MeV} / \mathrm{u}$ to $350 \mathrm{MeV} / \mathrm{u}$ in steps of $2 \mathrm{MeV} / \mathrm{u}$. The mean, standard deviation and maximum MRE between the calculated dose deposition profiles and the ground truth (obtained directly from the dose scored in the simulation) in the entrance, SOBP and tail regions for each simulated irradiation are presented without the use of regularisation in Table 1, and with the use of regularisation in Table 2. Examples of the parametric fitting of positron-emitting fragment profiles and estimated dose deposition profiles are presented in Figure 4 and 5 respectively, for 4 of the $14{ }^{12} \mathrm{C}$ SOBPs.

Finally, the distal edges of the SOBP dose profiles calculated using L1 regularisation were loeated for each of the $14{ }^{12} \mathrm{C}$ SOBP beams, and compared to the ground truth distal edge locations. The mean, standard deviation and maximum error between the calculated distal edge depth and ground truth are presented in Table 3. 


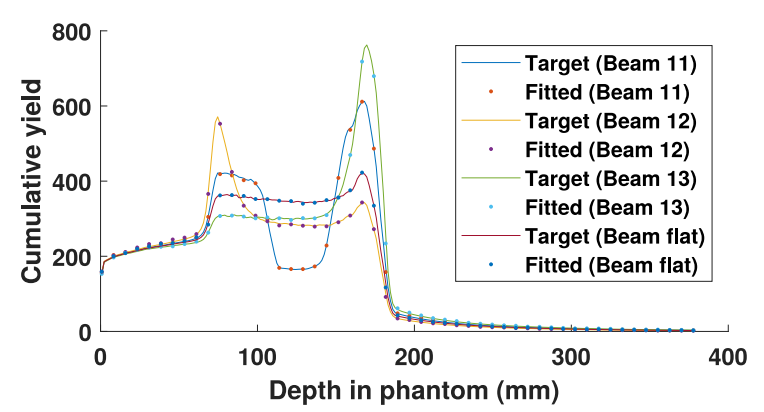

(a) ${ }^{11} \mathrm{C}$

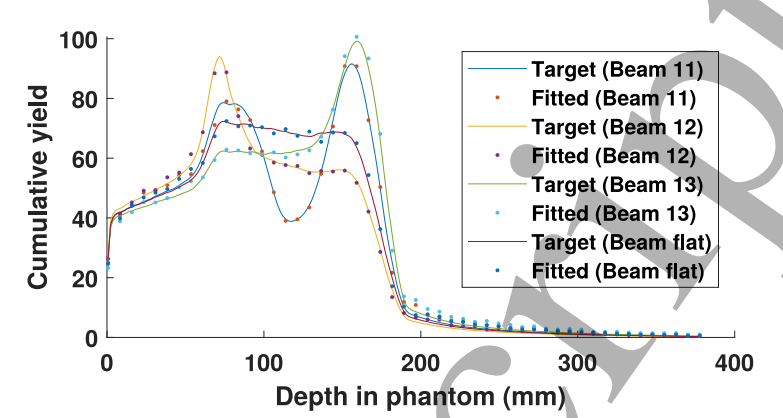

(b) ${ }^{10} \mathrm{C}$

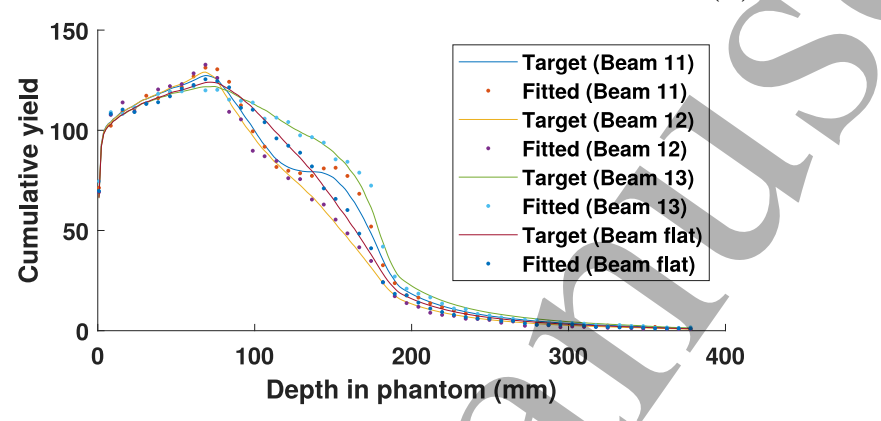

(c) ${ }^{15} \mathrm{O}$

Figure 4. Fragment profiles from four simulated polyenergetic ${ }^{12} \mathrm{C}$ beams, parametrically fitted using a library of fragment profiles from a range of monoenergetic incident beams. For clarity, only every fifth fitted point is shown.

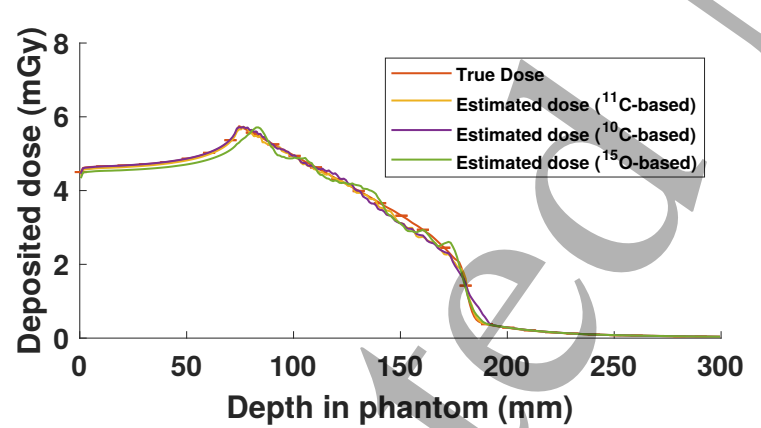

(a) Flat beam

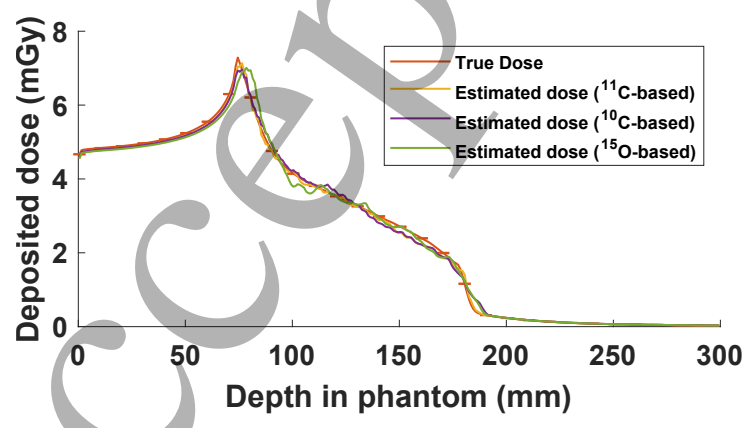

(c) Beam 12



(b) Beam 11

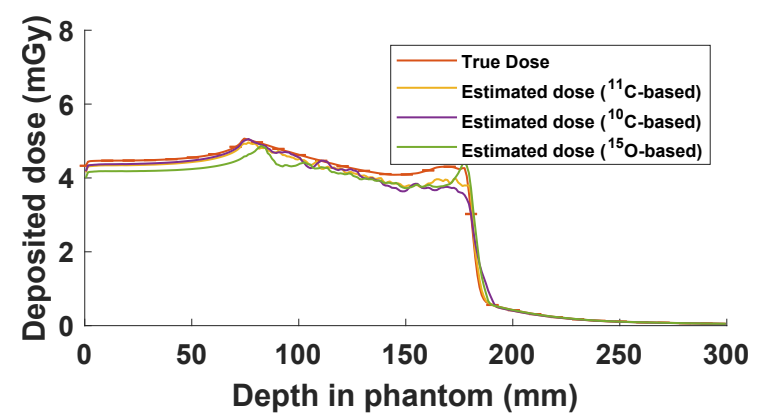

(d) Beam 13

Figure 5. Comparison of ground truth dose deposition profiles (red) of four simulated ${ }^{12} \mathrm{C}$ SOBPs, and dose profiles calculated using dose estimation procedure applied to the observed distributions of ${ }^{11} \mathrm{C}$ (yellow), ${ }^{10} \mathrm{C}$ (purple) and ${ }^{15} \mathrm{O}$ (green) fragments. L1 regularisation has been enforced to suppress high-frequency noise. 
Dose quantification in carbon ion therapy using in-beam PET

\begin{tabular}{lllll}
\hline & Region & Mean MRE (\%) & Std Dev. MRE (\%) & Max. MRE (\%) \\
\hline \multirow{2}{*}{${ }^{11} \mathrm{C}$} & Entrance & 1.0 & 0.9 & 3.3 \\
& SOBP & 1.7 & 0.9 & 4.2 \\
& Tail & 0.6 & 0.3 & 1.3 \\
& Overall & 0.9 & 0.5 & 2.3 \\
\hline \multirow{2}{*}{${ }^{10} \mathrm{C}$} & Entrance & 0.6 & 0.8 & 3.3 \\
& SOBP & 3.1 & 0.8 & 5.3 \\
& Tail & 1.7 & 0.5 & 2.8 \\
& Overall & 1.4 & 0.4 & 2.5 \\
\hline \multirow{2}{*}{$\mathrm{O}$ O } & Entrance & 1.7 & 1.5 & 6.2 \\
& SOBP & 4.7 & 0.8 & 6.8 \\
& Tail & 0.3 & 0.1 & 0.5 \\
& Overall & 2.3 & 0.6 & 4.1 \\
\hline
\end{tabular}

Table 1. Mean MRE, standard deviation of the MRE, and maximum MRE between calculated and ground truth dose profiles, without regularisation, over all SOBP ${ }^{12} \mathrm{C}$ beam deliveries.

\begin{tabular}{|c|c|c|c|c|}
\hline & Region & Mean MRE (\%) & Std Dev. MRE (\%) & Max. MRE (\%) \\
\hline \multirow{4}{*}{${ }^{11} \mathrm{C}$} & Entranc & 1.0 & 0.8 & 3.2 \\
\hline & SOBP & 1.5 & 0.9 & 3.9 \\
\hline & Tail & 0.7 & 0.3 & 1.4 \\
\hline & Overall & 0.8 & 0.5 & 2.2 \\
\hline \multirow{4}{*}{${ }^{10} \mathrm{C}$} & Entrance & 0.6 & 1.0 & 3.5 \\
\hline & SOBP & 2.1 & 1.0 & 5.0 \\
\hline & Tail & 1.0 & 0.4 & 1.6 \\
\hline & Overall & 1.0 & 0.6 & 2.4 \\
\hline 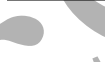 & Entrance & 1.8 & 1.5 & 6.3 \\
\hline${ }^{15} \mathrm{O}$ & SOBP & 3.1 & 1.1 & 6.0 \\
\hline 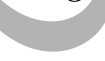 & Tail & 0.4 & 0.2 & 0.8 \\
\hline 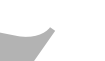 & Overall & 1.6 & 0.7 & 3.8 \\
\hline
\end{tabular}

Table 2. Mean MRE, standard deviation of the MRE, and maximum MRE between calculated and ground truth dose profiles, using $\mathrm{L} 1$ regularisation, over all $\mathrm{SOBP}{ }^{12} \mathrm{C}$ beam deliveries. 
Dose quantification in carbon ion therapy using in-beam PET

\begin{tabular}{llll}
\hline & Mean Error $(\mathrm{mm})$ & Std Dev. Error $(\mathrm{mm})$ & Max. Error $(\mathrm{mm})$ \\
\hline${ }^{11} \mathrm{C}$ & 0.7 & 0.8 & 2.0 \\
${ }^{10} \mathrm{C}$ & 1.9 & 1.7 & 4.0 \\
${ }^{15} \mathrm{O}$ & 1.2 & 1.3 & 3.0 \\
\hline
\end{tabular}

Table 3. Mean error, standard deviation of the error, and maximum error/between the distal edge of calculated and ground truth dose profiles, using L1 regularisation, over all SOBP ${ }^{12} \mathrm{C}$ beam deliveries.

\section{Discussion}

The mean overall MRE between calculated and true dose profiles remained below $0.9 \%$, $1.4 \%$ and $2.3 \%$ for ${ }^{11} \mathrm{C},{ }^{10} \mathrm{C}$ and ${ }^{15} \mathrm{O}$, without the use of $\mathrm{L} 1$ regularisation. After incorporating the regularisation term, the mean overall MREs were reduced to below $0.8 \%, 1.0 \%$ and $1.6 \%$ for ${ }^{11} \mathrm{C},{ }^{10} \mathrm{C}$ and ${ }^{15} \mathrm{O}$, respectively. The greatest errors after adding the regularisation term were observed when estimating the dose distribution based on ${ }^{15} \mathrm{O}$ fragments in the $\mathrm{SOBP}$ region.

The accuracy of dose estimation could potentially be further improved by implementing a combination of L1 and L2 regularisation, as L1 regularisation is typically better at preserving edge features, while L2 regularisation results in a better fit to smooth and continuous signals [45]. The scaling factor calculated to address the overestimation of yield profiles produced through the TAC-fitting procedure is expected to be dependent on the specific target material and image acquisition time. The factor is needed to compensate for the omission of the minor contributions of positron-emitting fragments other than ${ }^{11} \mathrm{C},{ }^{10} \mathrm{C}$ and ${ }^{15} \mathrm{O}$ from the model fitted to the total positron yield, and it is expected that the total yield of these fragments relative to ${ }^{11} \mathrm{C},{ }^{10} \mathrm{C}$ and ${ }^{15} \mathrm{O}$ will change in each target material, and therefore the factor required to correct for this error will change.

The distal edge of delivered SOBP dose profiles was reconstructed to within an average of $0.7 \mathrm{~mm}, 1.9 \mathrm{~mm}$ and $1.2 \mathrm{~mm}$ of the ground truth for ${ }^{11} \mathrm{C},{ }^{10} \mathrm{C}$ and ${ }^{15} \mathrm{O}$, respectively.

In this work, biological washout rates were not modeled since no biological processes are present to degrade the signal in a solid PMMA phantom. In reality, biological washout is a source of significant error in quantitative PET data analysis, and must be corrected for using a biological washout model [25, 29, 32]. By performing dose calculations using distributions of single fragment species, the PET scan length can be optimised for each isotope, depending on the biological washout rate in the target tissue. While the most accurate dose calculations were achieved using ${ }^{11} \mathrm{C}$ fragment distributions, the shorter half-life of ${ }^{10} \mathrm{C}$ allows for the dose quantification procedure to be implemented on data obtained from a relatively short PET scan, since more than $99 \%$ of ${ }^{10} \mathrm{C}$ fragments will have decayed within 2.5 minutes of the end of the irradiation period. In this case, a fast dose calculation may be performed using ${ }^{10} \mathrm{C}$ fragments 
based on a short initial PET scan to quickly identify any deviations from the treatment plan. However, in treatment regions where metabolic processes may rapidly affect the distribution of positron-emitting fragments, a washout model based on the washout rate of each significant positron-emitting fragment species should be applied $[25,32]$. A washout model will be incorporated in future in vivo dose estimation studies.

The present study is limited to the irradiation of a homogeneous PMMA target by polyenergetic ${ }^{12} \mathrm{C}$ ion beams. In order to extend this method to heterogeneous targets, including realistic tissue models, the basis function library must first be expanded to include fragment yield and deposited dose profiles for a range of homogeneous target materials. Heterogeneous targets will be divided into homogeneous sub-volumes, and acquired annihilation yield maps can be fitted using a piecewise function of fragment profiles from the expanded basis function library [60]. The material-dependent scaling factor used to correct the yield overestimation observed when separating the acquired dynamic yield profiles into each positron-emitting fragment species must be calculated for the material in each homogeneous sub-volume. Automatic selection of the proper correction factors can be performed using lookup tables based on automatically segmented CT or MRI image data. The factors will then be applied to the piecewise yield segments in their respective sub-volumes.

Finally, in this work, cumulative activity profiles were produced using Monte Carlo simulation of injected ${ }^{12} \mathrm{C}$ particles, where all particles were injected simultaneously. However, particle therapy is typically delivered in a series of consecutive spills [56]. The duration of each spill, and the duration of the beam-off period between each spill, will affect the cumulative activity profile as positron-emitting fragments will begin decaying immediately after their creation. In order to apply the dose quantification procedure to real therapeutic beam deliveries, additional terms must be included in (1) to account for the decay of positron-emitting fragments during the beam delivery. This will also enable inter-spill imaging, where PET data is acquired between consecutive spills while the beam is off.

During inter-spill periods, short-lived positron-emitting fragments, such as ${ }^{10} \mathrm{C}$, will decay at a greater rate than longer-lived positron-emitters, such as ${ }^{11} \mathrm{C}$. Consequently, despite the greater total yield of longer-lived positron-emitting fragments over a long scan length, short inter-spill images will be predominantly representative of short-lived positron-emitting fragment distributions. By using positron-emitting isotopes with short half-lives, such as ${ }^{10} \mathrm{C}$, real-time dose estimations based on inter-spill PET data will be feasible while mitigating the degrading effects of biological washout.

\section{Conclusion}

This work presented a dose quantification technique based on PET imaging in carbon ion therapy. A PET image of a volume irradiated by a polyenergetic ${ }^{12} \mathrm{C}$ beam is decomposed into the three main positron-emitting fragmentation products, and the proportional contribution of each energy component estimated by fitting a linear combination of 


\section{Dose quantification in carbon ion therapy using in-beam PET}

511 547 The accuracy of the profile interpolation procedure is assessed by splitting the set of 548

monoenergetic fragment profiles to the observed monoisotopic fragment distribution. The dose is then estimated by using the same weights to sum the individual dose profiles, subject to total variation regularisation, in each iteration of the fragment-fitting process. The method was evaluated by simulating a diverse range of polyenergetic ${ }^{12} \mathrm{C}$ firradiations of a homogeneous PMMA target using the Geant4 Monte Carlo toolkit, and comparing the estimated dose with the ground truth dose as measured directly in the simulation.

The proposed dose quantification method provided a very accurate estimate of the dose in the PMMA phantom, consistently yielding an estimated dose within $0.8 \%$ of the ground truth, and estimating the position of the distal edge of the SOBP to within $0.7 \mathrm{~mm}$ using the ${ }^{11} \mathrm{C}$ yield profile. For comparison, Hofmann's deconvolutionbased approach estimated the dose distribution from polyenergetic ${ }^{12} \mathrm{C}$ irradiation of a PMMA target from simulated PET images with an accuracy of $1.41 \%$, and estimated the distal edge position to within $0.25 \mathrm{~mm}$. Our work demonstrated that accurate dose estimates may be obtained even using short-lived positron-emitting fragments. The shorter half-lives of these fragments (particularly ${ }^{10} \mathrm{C}$ ) will allow dose estimates to be made after very short PET signal acquisitions, which will significantly reduce the impact of biological washout compared to a typical 20 minute post-irradiation scan. However, the limited extent to which biological washout has been studied in larger animals (including humans) to date means that this hypothesis cannot be evaluated via simulation and will require an in-vivo experiment to be performed to properly evaluate the benefits of short-acquisition scans in relation to washout.

Taking the idea of dosimetry via short-duration PET acquisitions to its ultimate conclusion, it may be possible that clinical dose estimates can be obtained in real time via inter-spill imaging targeting the distribution of short-lived positron-emitting isotopes, which would enable real-time adaptation of a treatment plan to correct for deviations from the planned dose distribution.

\section{Acknowledgements}

This research is supported by the Australian Government Research Training Program (AGRTP) scholarship and the AINSE Honours Scholarship Program. The authors would like to thank AINSE Limited for providing financial assistance (Award - Residential Student Scholarship 2018) to enable work on this research. This research was undertaken with the assistance of resources from the National Computational Infrastructure (NCI Australia), an NCRIS enabled capability supported by the Australian Government. This work was supported by the Multi-modal Australian ScienceS Imaging and Visualisation Environment (MASSIVE).

\section{Appendix A. Basis Function Generation using Profile Interpolation}


the monoenergetic irradiations of the PMMA target into a training set and testing set. The fragment and dose profiles of the $200 \mathrm{MeV} / \mathrm{u}, 230 \mathrm{MeV} / \mathrm{u}, 260 \mathrm{MeV} / \mathrm{u}$, $290 \mathrm{MeV} / \mathrm{u}, 320 \mathrm{MeV} / \mathrm{u}$, and $350 \mathrm{MeV} / \mathrm{u}$ monoenergetic beams are used to create a profile interpolation model; fragment and dose profiles for other 4 energies which have been simulated $(215 \mathrm{MeV} / \mathrm{u}, 245 \mathrm{MeV} / \mathrm{u}, 275 \mathrm{MeV} / \mathrm{u}$, and $305 \mathrm{MeV} / \mathrm{u})$ are then estimated using this profile interpolation model, and compared to the Monte Carlo simulated fragment and dose profiles. Comparisons of ground truth and estimated ${ }^{11} \mathrm{C}$, ${ }^{10} \mathrm{C}$ and ${ }^{15} \mathrm{O}$ fragment and dose profiles are shown in Figure A1. The MRE between true and estimated profiles in the entrance, SOBP and tail regions are presented in Table A1.

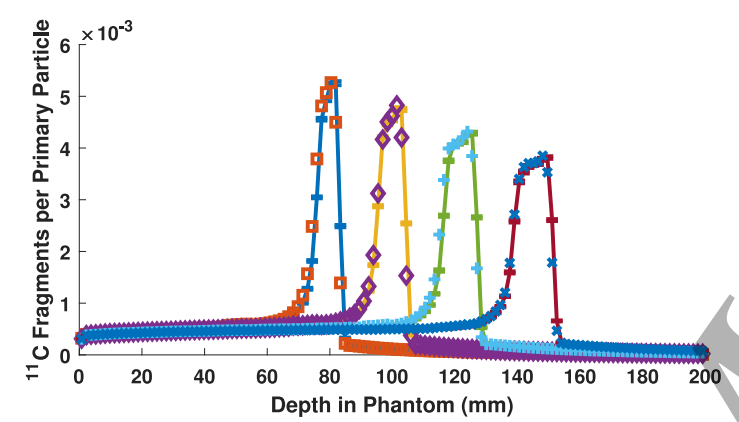

(a) ${ }^{11} \mathrm{C}$

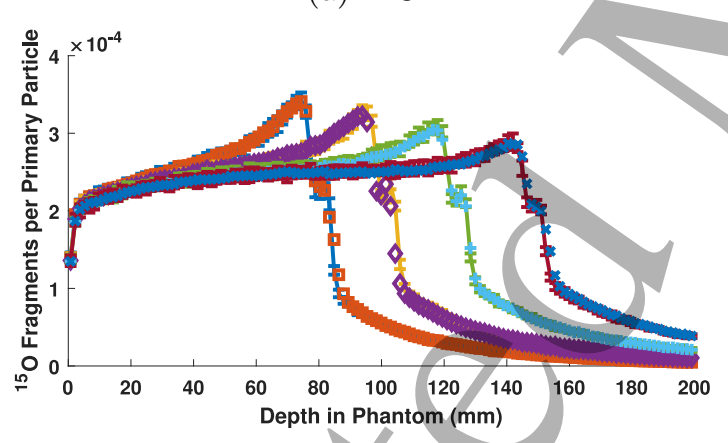

(c) ${ }^{15} \mathrm{O}$

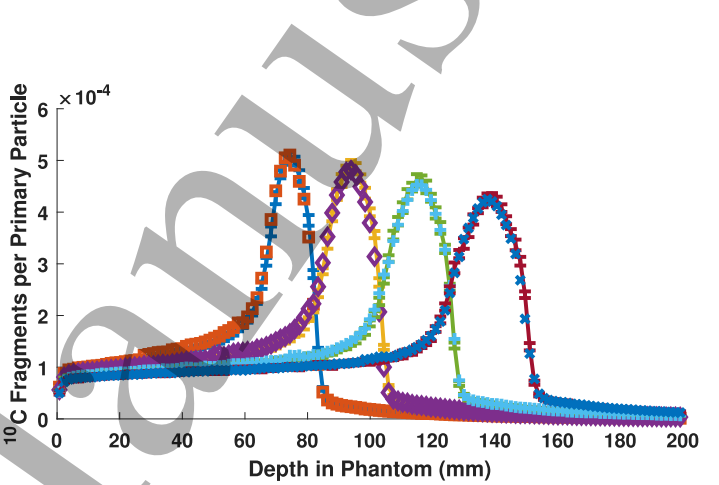

(b) ${ }^{10} \mathrm{C}$

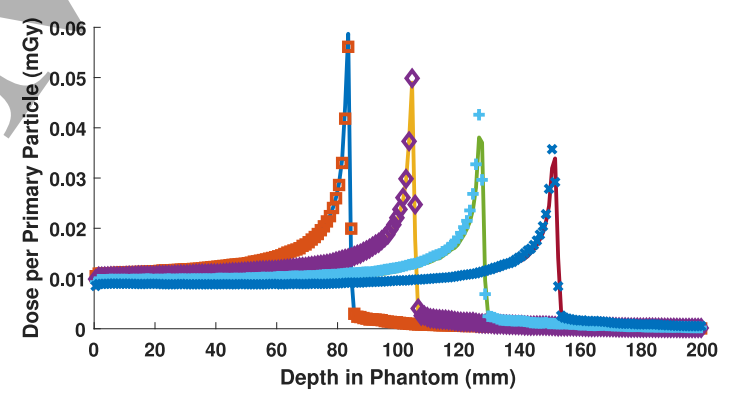

(d) Deposited dose

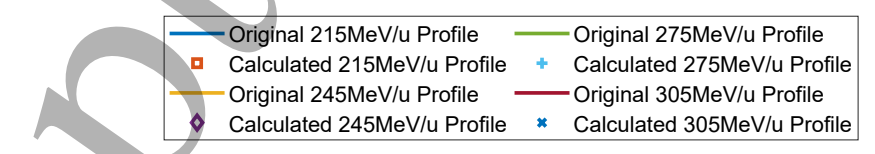

Figure A1. Profiles of ${ }^{11} \mathrm{C},{ }^{10} \mathrm{C}$, and ${ }^{15} \mathrm{O}$ fragment production and deposited dose profile resulting from irradiation of a homogeneous PMMA phantom by monoenergetic ${ }^{12} \mathrm{C}$ beams with energies of $215 \mathrm{MeV} / \mathrm{u}, 245 \mathrm{MeV} / \mathrm{u}, 275 \mathrm{MeV} / \mathrm{u}$ and $305 \mathrm{MeV} / \mathrm{u}$ in Monte Carlo simulation, plotted against fragment and dose profiles estimated using the profile interpolation procedure. 
Dose quantification in carbon ion therapy using in-beam PET

Table A1. Mean relative error between true and interpolated ${ }^{11} \mathrm{C},{ }^{10} \mathrm{C},{ }^{15} \mathrm{O}$ and deposited dose profiles, calculated in the entrance, SOBP and tail regions, relative to the maximum of the true profile in the specified region.

\begin{tabular}{|c|c|c|c|c|}
\hline \multirow{2}{*}{ Interpolated Profile } & \multirow{2}{*}{ Beam Energy $(\mathrm{MeV} / \mathrm{u})$} & \multicolumn{3}{|c|}{ Mean MRE (\%) } \\
\hline & & Entrance & SOBP & Tail \\
\hline \multirow{4}{*}{${ }^{11} \mathrm{C}$} & 215 & 0.49 & 5.81 & 0.41 \\
\hline & 245 & 0.51 & 4.04 & 0.41 \\
\hline & 275 & 0.92 & 5.07 & 0.37 \\
\hline & 305 & 1.01 & 4.19 & 0.48 \\
\hline \multirow{4}{*}{${ }^{10} \mathrm{C}$} & 215 & 0.39 & 1.57 & 0.44 \\
\hline & 245 & 0.59 & 2.71 & 0.65 \\
\hline & 275 & 0.55 & 4.11 & 0.78 \\
\hline & 305 & 0.52 & 2.69 & 0.93 \\
\hline \multirow{4}{*}{${ }^{15} \mathrm{O}$} & 215 & 0 & 3.11 & 0.42 \\
\hline & 245 & & 2.79 & 0.53 \\
\hline & 275 & 2 & 1.44 & 0.45 \\
\hline & 305 & 0.66 & 3.24 & 0.86 \\
\hline \multirow{4}{*}{ Dose } & 215 & 0.61 & 2.47 & 0.12 \\
\hline & 245 & 1.02 & 2.04 & 0.25 \\
\hline & 275 & 3.44 & 6.62 & 0.49 \\
\hline & & 3.00 & 5.67 & 0.66 \\
\hline
\end{tabular}

559

\section{Appendix B. Additional Information for ${ }^{12} \mathrm{C}$ SOBPs}

The weight values used in Monte Carlo simulation to deliver the flat ${ }^{12} \mathrm{C}$ SOBP, the $3{ }^{12} \mathrm{C}$ SOBPs containing well-defined dose maxima and/or minima, and $4{ }^{12} \mathrm{C}$ SOBPs with randomised weights are presented in Figure B1. 
Dose quantification in carbon ion therapy using in-beam PET

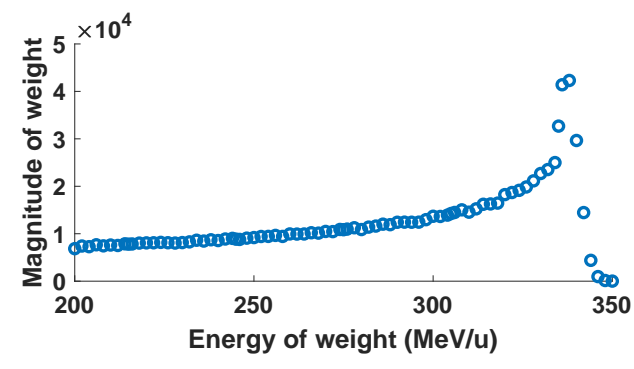

(a) Flat beam

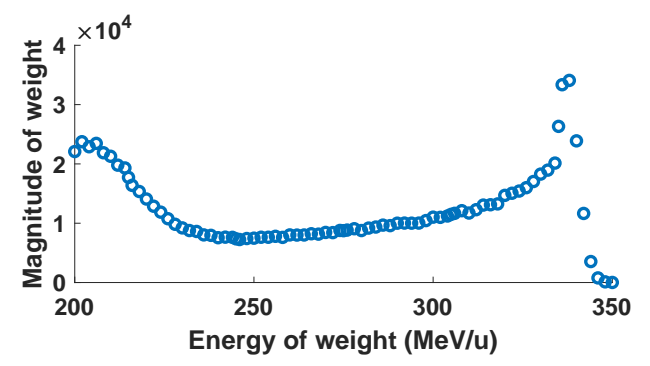

(c) Beam 12



(b) Beam 11

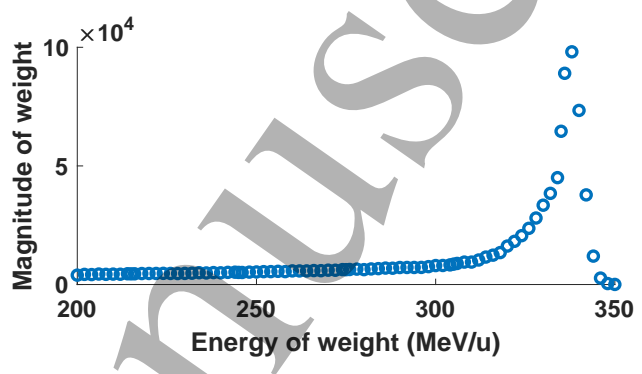

(d) Beam 13

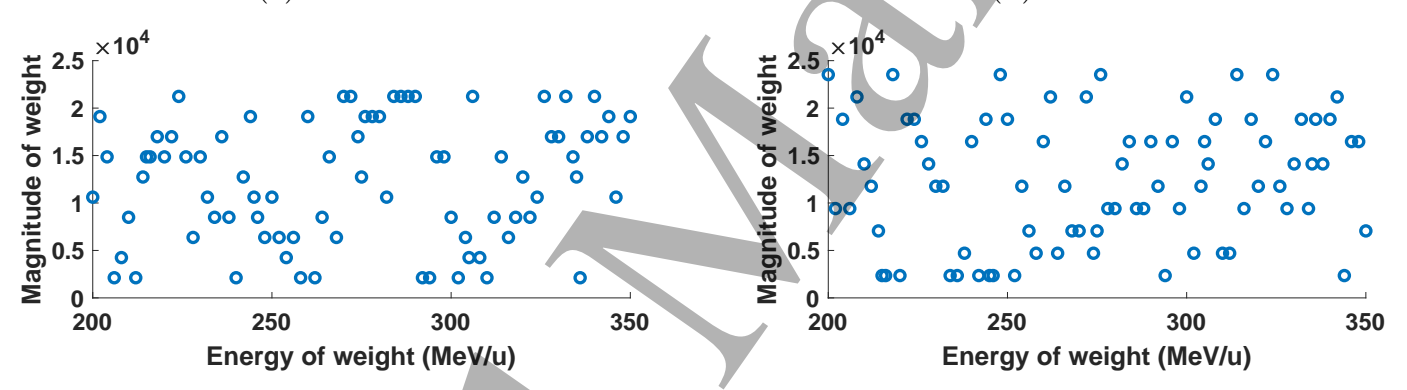

(e) Beam 1

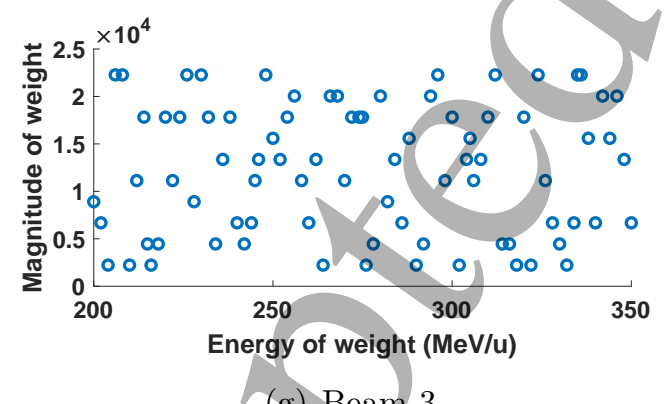

(g) Beam 3

(f) Beam 2

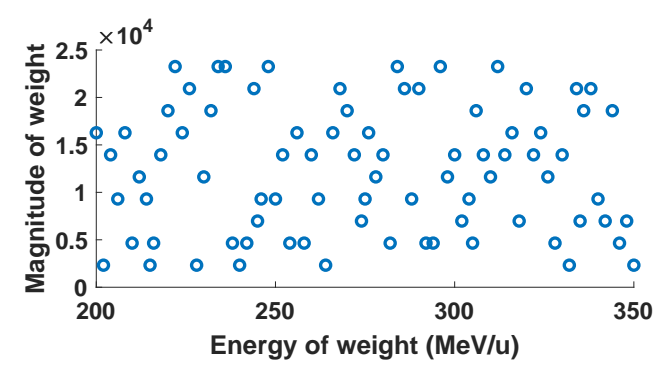

(h) Beam 4

Figure B1. Weight values used in Monte Carlo simulation to deliver a flat biological dose $(1(\mathrm{a}))$, the profiles with well-defined dose maxima and/or minima within the SOBP region (1(b)-1(d)), and 4 SOBPs produced using random weighting values (1(e)1(h)).

Examples of the positron-emitting fragment yield, and corresponding deposited 


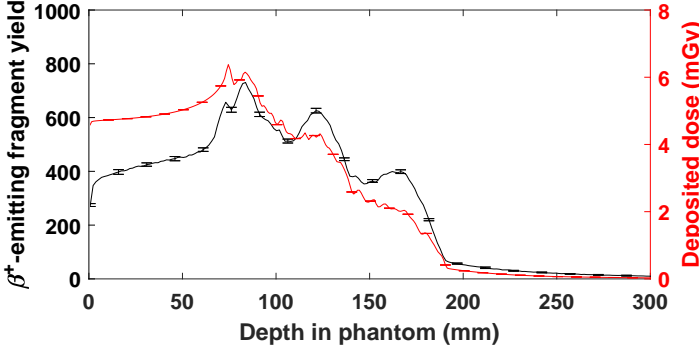

(a) Flat beam

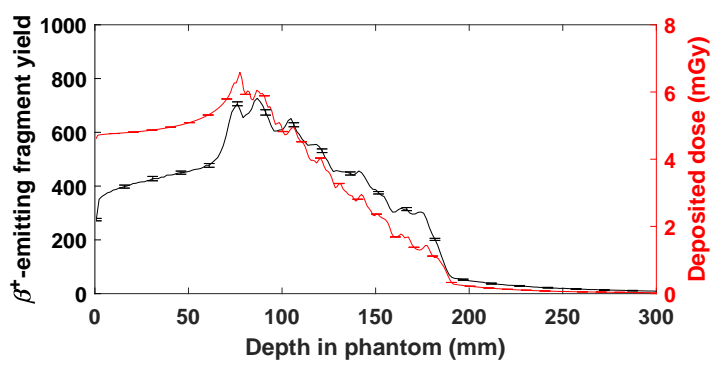

(c) Beam 12 (proximal maximum)

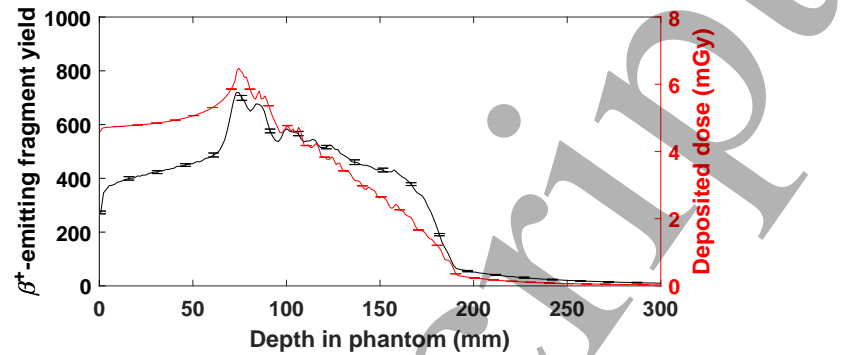

(b) Beam 11 (proximal and distal maxima)

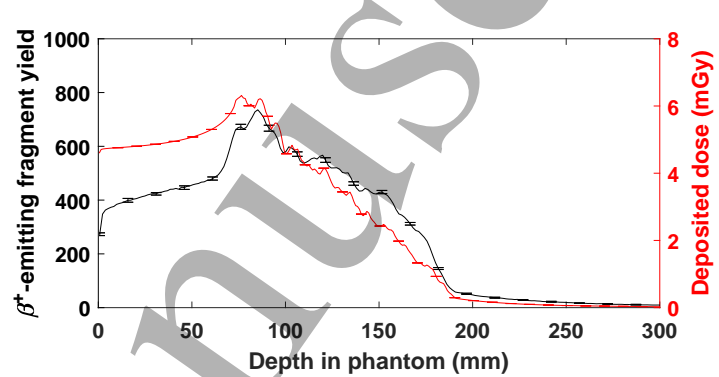

(d) Beam 13 (distal maximum)

Figure B2. Total positron-emitting fragment profiles (black) and deposited dose profiles (red) resulting from 4 of the 10 random-weighted ${ }^{12} \mathrm{C}$ SOBPs.

\section{References}

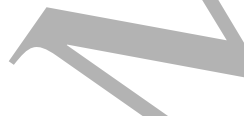

[1] O. Jäkel, D. Schulz-Ertner, C. Karger, A. Nikoghosyan, J. Debus, Heavy Ion Therapy: Status and Perspectives, Technology in Cancer Research \& Treatment 2 (5) (2003) 377-387 (Oct. 2003). doi:10.1177/153303460300200503. URL https://doi.org/10.1177/153303460300200503

[2] M. Durante, J. S. Loeffler, Charged particles in radiation oncology, Nature Reviews Clinical Oncology 7 (1) (2009) 37-43 (Dec. 2009). doi:10.1038/nrclinonc.2009.183. URL https://doi.org/10.1038/nrclinonc.2009.183

[3] M. Durante, R. Orecchia, J. S. Loeffler, Charged-particle therapy in cancer: clinical uses and future perspectives, Nature Reviews Clinical Oncology 14 (8) (2017) 483-495 (Mar. 2017). doi:10.1038/nrclinonc.2017.30.

URL https://doi.org/10.1038/nrclinonc.2017.30

[4] D. Schardt, T. Elsässer, D. Schulz-Ertner, Heavy-ion tumor therapy: Physical and radiobiological benefits, Reviews of Modern Physics 82 (1) (2010) 383-425 (Feb. 2010). doi:10.1103/revmodphys.82.383.

URL https://doi.org/10.1103/revmodphys.82.383

[5] M. Durante, H. Paganetti, Nuclear physics in particle therapy: a review, Reports on Progress in Physics 79 (9) (2016) 096702 (Aug. 2016). doi:10.1088/0034-4885/79/9/096702.

URL https://doi.org/10.1088/0034-4885/79/9/096702

[6] U. Schneider, E. Pedroni, A. Lomax, The calibration of CT Hounsfield units for radiotherapy treatment planning, Physics in Medicine and Biology 41 (1) (1996) 111-124 (Jan. 1996). doi:10.1088/0031-9155/41/1/009.

URL https://doi.org/10.1088/0031-9155/41/1/009

[7] G. Kraft, U. Arndt, W. Becher, D. Schardt, H. Stelzer, U. Weber, T. Archinal, Heavy ion therapy at GSI, Nuclear Instruments and Methods in Physics Research Section A: Accelerators, Spectrometers, Detectors and Associated Equipment 367 (1-3) (1995) 66-70 (Dec. 1995). 
Dose quantification in carbon ion therapy using in-beam PET

doi:10.1016/0168-9002(95)00735-0.

URL https://doi.org/10.1016/0168-9002(95)00735-0

[8] Y. Iseki, H. Mizuno, Y. Futami, T. Tomitani, T. Kanai, M. Kanazawa, A. Kitagawa, T. Murakami, T. Nishio, M. Suda, E. Urakabe, A. Yunoki, H. Sakai, Positron camera for range verification of heavy-ion radiotherapy, Nuclear Instruments and Methods in Physics Research Section A: Accelerators, Spectrometers, Detectors and Associated Equipment 515 (3) (2003) 840-849 (Dec. 2003). doi:10.1016/j.nima.2003.07.005.

URL https://doi.org/10.1016/j.nima.2003.07.005

[9] K. Parodi, H. Paganetti, H. A. Shih, S. Michaud, J. S. Loeffler, T. F. DeLaney, N. J. Liebsch, J. E. Munzenrider, A. J. Fischman, A. Knopf, T. Bortfeld, Patient Study of In Vivo Verification of Beam Delivery and Range, Using Positron Emission Tomography and Computed Tomography Imaging After Proton Therapy, International Journal of Radiation Oncology, Biology, Physics 68 (3) (2007) 920-934 (Jul. 2007). doi:10.1016/j.ijrobp.2007.01.063.

URL https://doi.org/10.1016/j.ijrobp.2007.01.063

[10] S. E. Combs, J. Bauer, D. Unholtz, C. Kurz, T. Welzel, D. Habermehl, T. Haberer, J. Debus, K. Parodi, Monitoring of patients treated with particle therapy using positron-emissiontomography (PET): the MIRANDA study, BMC Cancer 12 (1) (Apr. 2012). doi:10.1186/14712407-12-133. URL https://doi.org/10.1186/1471-2407-12-133

[11] E. Yoshida, H. Tashima, T. Shinaji, K. Shimizu, H. Wakizaka, A. Mohammadi, F. Nishikido, T. Yamaya, Development of a Whole-Body Dual Ring OpenPET for in-Beam PET, IEEE Transactions on Radiation and Plasma Medical Sciences 1 (4) (2017) 293-300 (Jul. 2017). doi:10.1109/trpms.2017.2703823. URL https://doi.org/10.1109/trpms.2017.2703823

[12] J. Pawelke, L. Byars, W. Enghardt, W. D. Fromm, H. Geissel, B. G. Hasch, K. Lauckner, P. Manfraß, D. Schardt, M. Sobiella, The investigation of different cameras for in-beam PET imaging, Physics in Medicine \& Biology 41 (2) (1996) 279-296 (Feb. 1996). doi:10.1088/00319155/41/2/006.

URL https://doi.org/10.1088/0031-9155/41/2/006

[13] G. Sportelli, N. Belcari, N. Camarlinghi, G. A. P. Cirrone, G. Cuttone, S. Ferretti, A. Kraan, J. E. Ortuño, F. Romano, A. Santos, K. Straub, A. Tramontana, A. D. Guerra, V. Rosso, First fullbeam PET acquisitions in proton therapy with a modular dual-head dedicated system, Physics in Medicine \& Biology 59 (1) (2013) 43-60 (Dec. 2013). doi:10.1088/0031-9155/59/1/43. URL https://doi.org/10.1088/0031-9155/59/1/43

[14] Y. Shao, X. Sun, K. Lou, X. R. Zhu, D. Mirkovic, F. Poenisch, D. Grosshans, In-beam PET imaging for on-line adaptive proton therapy: an initial phantom study, Physics in Medicine \& Biology 59 (13) (2014) 3373-3388 (May 2014). doi:10.1088/0031-9155/59/13/3373. URL https://doi.org/10.1088/0031-9155/59/13/3373

[15] K. Parodi, Vision 20/20: Positron emission tomography in radiation therapy planning, delivery, and monitoring, Medical Physics 42 (12) (2015) 7153-7168 (Dec. 2015). doi:10.1118/1.4935869. URL https://doi.org/10.1118/1.4935869

[16] M. G. Bisogni, A. Attili, G. Battistoni, N. Belcari, N. Camarlinghi, P. Cerello, S. Coli, A. D. Guerra, A. Ferrari, V. Ferrero, E. Fiorina, G. Giraudo, E. Kostara, M. Morrocchi, F. Pennazio, C. Peroni, M. A. Piliero, G. Pirrone, A. Rivetti, M. D. Rolo, V. Rosso, P. Sala, G. Sportelli, R. Wheadon, INSIDE in-beam positron emission tomography system for particle range monitoring in hadrontherapy, Journal of Medical Imaging 4 (1) (2016) 011005 (Dec. 2016). doi:10.1117/1.jmi.4.1.011005. URL https://doi.org/10.1117/1.jmi.4.1.011005

[17] P. C. Lopes, J. Bauer, A. Salomon, I. Rinaldi, V. Tabacchini, T. Tessonnier, P. Crespo, K. Parodi, D. R. Schaart, Firstin situTOF-PET study using digital photon counters for proton range verification, Physics in Medicine \& Biology 61 (16) (2016) 6203-6230 (Aug. 2016). 
Dose quantification in carbon ion therapy using in-beam PET

doi:10.1088/0031-9155/61/16/6203.

URL https://doi.org/10.1088/0031-9155/61/16/6203

[18] H. Tashima, E. Yoshida, N. Inadama, F. Nishikido, Y. Nakajima, H. Wakizaka, T. Shinaji, M. Nitta, S. Kinouchi, M. Suga, H. Haneishi, T. Inaniwa, T. Yamaya, Development of a small single-ring OpenPET prototype with a novel transformable architecture, Physics in Medicine \& Biology 61 (4) (2016) 1795-1809 (Feb. 2016). doi:10.1088/0031-9155/61/4/1795. URL https://doi.org/10.1088/0031-9155/61/4/1795

[19] A. Paans, J. Schippers, Proton therapy in combination with PET as monitor: a feasibility study, IEEE Transactions on Nuclear Science 40 (4) (1993) 1041-1044 (Aug. 1993). doi:10.1109/23.256709.

URL https://doi.org/10.1109/23.256709

[20] Y. Hishikawa, K. Kagawa, M. Murakami, H. Sakai, T. Akagi, M. Abe, Usefulness of positronemission tomographic images after proton therapy, International Journal of Radiation Oncology, Biology, Physics 53 (5) (2002) 1388-1391 (Aug. 2002). doi:10.1016/s0360-3016(02)02887-0. URL https://doi.org/10.1016/s0360-3016(02) 02887-0

[21] T. Nishio, T. Sato, H. Kitamura, K. Murakami, T. Ogino, Distributions of $\beta^{+}$decayed nuclei generated in the $\mathrm{CH} 2$ and $\mathrm{H} 2 \mathrm{O}$ targets by the target nuclear fragment reaction using therapeutic MONO and SOBP proton beam, Medical Physics 32 (4) (2005) 1070-1082 (Mar. 2005). doi:10.1118/1.1879692. URL https://doi.org/10.1118/1.1879692

[22] C. Ma, T. Lomax, W. Hendee, Proton and Carbon Ion Therapy, Taylor and Francis Group, 2013 (2013).

[23] W. Enghardt, K. Parodi, P. Crespo, F. Fiedler, J. Pawelke, F. Pönisch, Dose quantification from in-beam positron emission tomography, Radiotherapy and Oncology 73 (2004) S96-S98 (Dec. 2004). doi:10.1016/s0167-8140(04)80024-0. URL https://doi.org/10.1016/s0167-8140(04)80024-0

[24] K. Frey, J. Bauer, D. Unholtz, C. Kurz, M. Krämer, T. Bortfeld, K. Parodi, TPSPET — A TPSbased approach for in vivo dose verification with PET in proton therapy, Physics in Medicine \& Biology 59 (1) (2013) 1-21 (Dec. 2013). doi:10.1088/0031-9155/59/1/1. URL https://doi.org/10.1088/0031-9155/59/1/1

[25] C. Ammar, K. Frey, J. Bauer, C. Melzig, S. Chiblak, M. Hildebrandt, D. Unholtz, C. Kurz, S. Brons, J. Debus, A. Abdollahi, K. Parodi, Comparing the biological washout of $\beta^{+}$-activity induced in mice brain after 12C-ion and proton irradiation, Physics in Medicine \& Biology 59 (23) (2014) 7229-7244 (Nov. 2014). doi:10.1088/0031-9155/59/23/7229. URL https://doi.org/10/1088/0031-9155/59/23/7229

[26] M. Priegnitz, D. Möckel, K. Parodi, F. Sommerer, F. Fiedler, W. Enghardt, In-beam PET measurement of ${ }^{7} \mathrm{Li}^{3+}$ irradiation induced $\beta^{+}$-activity, Physics in Medicine \& Biology 53 (16) (2008) 4443-4453 (Jul, 2008). doi:10.1088/0031-9155/53/16/015. URL https://doi.org/10.1088/0031-9155/53/16/015

[27] H. Mizuno, T. Tomitani, M. Kanazawa, A. Kitagawa, J. Pawelke, Y. Iseki, E. Urakabe, M. Suda, A. Kawano, R. Iritani, S. Matsushita, T. Inaniwa, T. Nishio, S. Furukawa, K. Ando, Y. K. Nakamura, T. Kanai, K. Ishii, Washout measurement of radioisotope implanted by radioactive beams in the rabbit, Physics in Medicine \& Biology 48 (15) (2003) 2269-2281 (Jul. 2003). doi:10.1088/0031-9155/48/15/302. URL https://doi.org/10.1088/0031-9155/48/15/302

[28] T. Tomitani, J. Pawelke, M. Kanazawa, K. Yoshikawa, K. Yoshida, M. Sato, A. Takami, M. Koga, Y. Futami, A. Kitagawa, E. Urakabe, M. Suda, H. Mizuno, T. Kanai, H. Matsuura, I. Shinoda, S. Takizawa, Washout studies of $11 \mathrm{C}$ in rabbit thigh muscle implanted by secondary beams of HIMAC, Physics in Medicine \& Biology 48 (7) (2003) 875-889 (Mar. 2003). doi:10.1088/00319155/48/7/305. 
Dose quantification in carbon ion therapy using in-beam PET

[29] I. Martínez-Rovira, C. Jouvie, S. Jan, Technical Note: Implementation of biological washout processes within GATE/GEANT4-A Monte Carlo study in the case of carbon therapy treatments, Medical Physics 42 (4) (2015) 1773-1778 (Mar. 2015). doi:10.1118/1.4914449. URL https://doi.org/10.1118/1.4914449

[30] Y. Hirano, S. Kinouchi, Y. Ikoma, E. Yoshida, H. Wakizaka, H. Ito, T. Yamaya, Compartmental analysis of washout effect in rat brain: in-beam OpenPET measurement using a 11C beam, Physics in Medicine \& Biology 58 (23) (2013) 8281-8294 (Nov. 2013). doi:10.1088/00319155/58/23/8281. URL https://doi.org/10.1088/0031-9155/58/23/8281

[31] Y. Hirano, H. Takuwa, E. Yoshida, F. Nishikido, Y. Nakajima, H. Wakizaka, T. Yamaya, Washout rate in rat brain irradiated by a $11 \mathrm{C}$ beam after acetazolamide loading using a small singlering OpenPET prototype, Physics in Medicine \& Biology 61 (5) (2016) 1875-1887 (Feb. 2016). doi:10.1088/0031-9155/61/5/1875. URL https://doi.org/10.1088/0031-9155/61/5/1875

[32] C. Toramatsu, E. Yoshida, H. Wakizaka, A. Mohammadi, Y. Ikoma, H. Tashima, F. Nishikido, A. Kitagawa, K. Karasawa, Y. Hirano, T. Yamaya, Washout effect in rabbit brain: in-beam PET measurements using 10C, $11 \mathrm{C}$ and $15 \mathrm{O}$ ion beams, Biomedical Physics \& Engineering Express 4 (3) (2018) 035001 (Mar. 2018). doi:10.1088/2057-1976/aaade7. URL https://doi.org/10.1088/2057-1976/aaade7

[33] A.-C. Knopf, K. Parodi, H. Paganetti, T. Bortfeld, J. Daartz, M. Engelsman, N. Liebsch, H. Shih, Accuracy of Proton Beam Range Verification Using Post-Treatment Positron Emission Tomography/Computed Tomography as Function of Treatment Site, International Journal of Radiation Oncology, Biology, Physics 79 (1) (2011) 297-304 (Jan. 2011). doi:10.1016/j.ijrobp.2010.02.017. URL https://doi.org/10.1016/j.ijrobp.2010.02.017

[34] A. C. Kraan, Range Verification Methods in Particle Therapy: Underlying Physics and Monte Carlo Modeling, Frontiers in Oncology 5 (Jul. 2015). doi:10.3389/fonc.2015.00150.

URL https://doi.org/10.3389/fonc.2015.00150

[35] S. Agostinelli, J. Allison, K. Amako, J. Apostolakis, H. Araujo, P. Arce, M. Asai, D. Axen, S. Banerjee, G. Barrand, F. Behner, L. Bellagamba, J. Boudreau, L. Broglia, A. Brunengo, H. Burkhardt, S. Chauvie, J. Chuma, R. Chytracek, G. Cooperman, G. Cosmo, P. Degtyarenko, A. Dell'Acqua, G. Depaola, D. Dietrich, R. Enami, A. Feliciello, C. Ferguson, H. Fesefeldt, G. Folger, F. Foppiano, A. Forti, S. Garelli, S. Giani, R. Giannitrapani, D. Gibin, J. G. Cadenas, I. González, G. G. Abril, G. Greeniaus, W. Greiner, V. Grichine, A. Grossheim, S. Guatelli, P. Gumplinger, R. Hamatsu, K. Hashimoto, H. Hasui, A. Heikkinen, A. Howard, V. Ivanchenko, A. Johnson, F. Jones, J. Kallenbach, N. Kanaya, M. Kawabata, Y. Kawabata, M. Kawaguti, S. Kelner, P. Kent, A. Kimura, T. Kodama, R. Kokoulin, M. Kossov, H. Kurashige, E. Lamanna, T. Lampén, V. Lara, V. Lefebure, F. Lei, M. Liendl, W. Lockman, F. Longo, S. Magni, M. Maire, E. Medernach, K. Minamimoto, P. M. de Freitas, Y. Morita, K. Murakami, M. Nagamatu, R. Nartallo, P. Nieminen, T. Nishimura, K. Ohtsubo, M. Okamura, S. O'Neale, Y. Oohata, K. Paech, J. Perl, A. Pfeiffer, M. Pia, F. Ranjard, A. Rybin, S. Sadilov, E. D. Salvo, G. Santin, T. Sasaki, N. Savvas, Y. Sawada, S. Scherer, S. Sei, V. Sirotenko, D. Smith, N. Starkov, H. Stoecker, J. Sulkimo, M. Takahata, S. Tanaka, E. Tcherniaev, E. S. Tehrani, M. Tropeano, P. Truscott, H. Uno, L. Urban, P. Urban, M. Verderi, A. Walkden, W. Wander, H. Weber, J. Wellisch, T. Wenaus, D. Williams, D. Wright, T. Yamada, H. Yoshida, D. Zschiesche, Geant4-a simulation toolkit, Nuclear Instruments and Methods in Physics Research Section A: Accelerators, Spectrometers, Detectors and Associated Equipment 506 (3) (2003) 250-303 (Jul. 2003). doi:10.1016/s0168-9002(03)01368-8. URL https://doi.org/10.1016/s0168-9002(03)01368-8

[36] K. Parodi, On the feasibility of dose quantification with in-beam PET data in radiotherapy with $12 \mathrm{C}$ and proton beams, Dissertation, Forschungszentrum Rossendorf (Nov. 2004). 
Dose quantification in carbon ion therapy using in-beam PET

[37] K. Parodi, T. Bortfeld, A filtering approach based on Gaussian-powerlaw convolutions for local PET verification of proton radiotherapy, Physics in Medicine \& Biology 51 (8) (2006) 1991-2009 (Mar. 2006). doi:10.1088/0031-9155/51/8/003. URL https://doi.org/10.1088/0031-9155/51/8/003

[38] F. Attanasi, A. Knopf, K. Parodi, H. Paganetti, T. Bortfeld, V. Rosso, A. D. Guerra, Extension and validation of an analytical model for in vivo PET verification of proton therapy - a phantom and clinical study, Physics in Medicine \& Biology 56 (16) (2011) 5079-5098 (Jul. 2011). doi:10.1088/0031-9155/56/16/001. URL https://doi.org/10.1088/0031-9155/56/16/001

[39] M. Pinto, K. Kroeniger, J. Bauer, R. Nilsson, E. Traneus, K. Parodi, A filtering approach for PET and PG predictions in a proton treatment planning system, Physics in Medicine and Biology [in press] (Mar. 2020). doi:10.1088/1361-6560/ab8146. URL https://doi.org/10.1088/1361-6560/ab8146

[40] A. Knopf, K. Parodi, H. Paganetti, E. Cascio, A. Bonab, T. Bortfeld, Quantitative assessment of the physical potential of proton beam range verification with PET/CT, Physics in Medicine \& Biology 53 (15) (2008) 4137-4151 (Jul. 2008). doi:10.1088/0031-9155/53/15/009. URL https://doi.org/10.1088/0031-9155/53/15/009

[41] F. Attanasi, N. Belcari, M. Camarda, A. D. Guerra, S. Moehrs, V. Rosso, S. Vecchio, N. Lanconelli, G. Cirrone, F. D. Rosa, G. Russo, Experimental validation of the filtering approach for dose monitoring in proton therapy at low energy, Physica Medica 24 (2) (2008) 102-106 (Jun. 2008). doi:10.1016/j.ejmp.2008.03.001. URL https://doi.org/10.1016/j.ejmp.2008.03.001

[42] T. Nishio, A. Miyatake, T. Ogino, K. Nakagawa, N. Saijo, H. Esumi, The development and clinical use of a beam on-line pet system mounted on a rotating gantry port in proton therapy, International Journal of Radiation Oncology, Biology, Physics 76 (1) (2010) 277-286 (Jan. 2010). doi:10.1016/j.ijrobp.2009.05.065. URL https://doi.org/10.1016/j.ijrobp,2009.05.065

[43] M. Aiello, F. Attanasi, N. Belcari, V. Rosso, K. Straub, A. D. Guerra, A Dose Determination Procedure by PET Monitoring in Proton Therapy: Monte Carlo Validation, IEEE Transactions on Nuclear Science 60 (5) (2013) 3298-3304 (Oct. 2013). doi:10.1109/tns.2013.2266734. URL https://doi.org/10.1109/tns.2013.2266734

[44] T. Hofmann, A. Fochi, K. Parodi, M. Pinto, Prediction of positron emitter distributions for range monitoring in carbon ion therapy: an analytical approach, Physics in Medicine \& Biology (Apr. 2019). doi:10.1088/1361-6560/ab17f9. URL https://doi.org/10/1088/1361-6560/ab17f9

[45] S. Remmele, J. Hesser, H. Paganetti, T. Bortfeld, A deconvolution approach for PET-based dose reconstruction in proton radiotherapy, Physics in Medicine \& Biology 56 (23) (2011) 7601-7619 (Nov. 2011). doi:10.1088/0031-9155/56/23/017.

URL https://doi.org/10.1088/0031-9155/56/23/017

[46] A. Ferrari, P, Sala, A. Fasso, J. Ranft, A Multi-Particle Transport Code (Program version 2005), Tech. rep., CERN-2005-010, SLAC-R-773, INFN-TC-05-11 (Oct. 2005).

[47] E. Fourkal, I. Velchev, J. Fan, W. Luo, C.-M. Ma, Energy optimization procedure for treatment planning with laser-accelerated protons, Medical Physics 34 (2) (2007) 577-584 (Jan. 2007). doi:10.1118/1.2431424. URL https://doi.org/10.1118/1.2431424

[48] E. Fourkal, J. Fan, I. Veltchev, Absolute dose reconstruction in proton therapy using PET imaging modality: feasibility study, Physics in Medicine \& Biology 54 (11) (2009) N217-N228 (May 2009). doi:10.1088/0031-9155/54/11/n02. URL https://doi.org/10.1088/0031-9155/54/11/n02

[49] T. Hofmann, M. Pinto, A. Mohammadi, M. Nitta, F. Nishikido, Y. Iwao, H. Tashima, E. Yoshida, A. Chacon, M. Safavi-Naeini, A. Rosenfeld, T. Yamaya, K. Parodi, Dose reconstruction from 
Dose quantification in carbon ion therapy using in-beam PET

PET images in carbon ion therapy: a deconvolution approach, Physics in Medicine \& Biology 64 (2) (2019) 025011 (Jan. 2019). doi:10.1088/1361-6560/aaf676.

URL https://doi.org/10.1088/1361-6560/aaf676

[50] T. Inaniwa, T. Kohno, T. Tomitani, Simulation for position determination of distal and proximal edges for SOBP irradiation in hadron therapy by using the maximum likelihood estimation method, Physics in Medicine \& Biology 50 (24) (2005) 5829-5845 (Dec. 2005). doi:10.1088/0031$9155 / 50 / 24 / 005$. URL https://doi.org/10.1088/0031-9155/50/24/005

[51] T. Inaniwa, T. Kohno, T. Tomitani, S. Sato, Monitoring the irradiation field of $12 \mathrm{C}$ and16O SOBP beams using positron emitters produced through projectile fragmentation reactions, Physics in Medicine \& Biology 53 (3) (2008) 529-542 (Jan. 2008). doi:10.1088/0031-9155/53/3/002. URL https://doi.org/10.1088/0031-9155/53/3/002

[52] T. Masuda, T. Nishio, J. Kataoka, M. Arimoto, A. Sano, K. Karasawa, ML-EM algorithm for dose estimation using PET in proton therapy, Physics in Medicine \& Biology (Jul. 2019). doi:10.1088/1361-6560/ab3276. URL https://doi.org/10.1088/1361-6560/ab3276

[53] T. Sato, Y. Iwamoto, S. Hashimoto, T. Ogawa, T. Furuta, S. ichiro Abe, T. Kai, P.-E. Tsai, N. Matsuda, H. Iwase, N. Shigyo, L. Sihver, K. Niita, Features of Particle and Heavy Ion Transport code System (PHITS) version 3.02, Journal of Nuclear Science and Technology 55 (6) (2018) 684-690 (Jan. 2018). doi:10.1080/00223131.2017.1419890. URL https://doi.org/10.1080/00223131.2017.1419890

[54] Geant4 Physics Reference Manual.

[55] A. Chacon, S. Guatelli, H. Rutherford, D. Bolst, A. Mohammadi, A. Ahmed, M. Nitta, F. Nishikido, Y. Iwao, H. Tashima, E. Yoshida, G. Akamatsu, S. Takyu, A. Kitagawa, T. Hofmann, M. Pinto, D. R. Franklin, K. Parodi, T. Yamaya, A. Rosenfeld, M. Safavi-Naeini, Comparative study of alternative Geant4 hadronic ion inelastic physics models for prediction of positron-emitting radionuclide production in carbon and oxygen ion therapy, Physics in Medicine \& Biology 64 (15) (2019) 155014 (Aug. 2019). doi:10.1088/1361-6560/ab2752. URL https://doi.org/10.1088/1361-6560/ab2752

[56] A. Mohammadi, E. Yoshida, H. Tashima, F. Nishikido, T. Inaniwa, A. Kitagawa, T. Yamaya, Production of an ${ }^{15} \mathrm{O}$ beam using a stable oxygen ion beam for in-beam PET imaging, Nuclear Instruments and Methods in Physics Research Section A: Accelerators, Spectrometers, Detectors and Associated Equipment 849 (2017) 76-82 (Mar. 2017). doi:10.1016/j.nima.2016.12.028. URL https://doi.org/10.1016/j.nima.2016.12.028

[57] G. Akamatsu, H. Tashima, Y. Iwao, H. Wakizaka, T. Maeda, A. Mohammadi, S. Takyu, M. Nitta, F. Nishikido, H. Rutherford, A. Chacon, M. Safavi-Naeini, E. Yoshida, T. Yamaya, Performance evaluation of a whole-body prototype PET scanner with four-layer DOI detectors, Physics in Medicine and Biology 64 (9) (2019) 095014 (Apr. 2019). doi:10.1088/1361-6560/ab18b2. URL https://doi.org/10.1088/1361-6560/ab18b2

[58] D. R. White, J. Booz, R. V. Griffith, J. J. Spokas, I. J. Wilson, Report 44, Journal of the International Commission on Radiation Units and Measurements os23 (1) (1989) NP-NP (Jan. 1989). doi:10.1093/jicru/os23.1.report44.

URL https://doi.org/10.1093/jicru/os23.1.report44

[59] A. Chacon, M. Safavi-Naeini, D. Bolst, S. Guatelli, D. R. Franklin, Y. Iwao, G. Akamatsu, H. Tashima, E. Yoshida, F. Nishikido, A. Kitagawa, A. Mohammadi, M.-C. Gregoire, T. Yamaya, A. B. Rosenfeld, Monte Carlo investigation of the characteristics of radioactive beams for heavy ion therapy, Scientific Reports 9 (1) (Apr. 2019). doi:10.1038/s41598-019-43073-1. URL https : //doi .org/10.1038/s41598-019-43073-1

[60] M. Priegnitz, F. Fiedler, D. Kunath, K. Laube, W. Enghardt, An experiment-based approach for predicting positron emitter distributions produced during therapeutic ion irradiation, IEEE Transactions on Nuclear Science 59 (1) (2012) 77-87 (Feb. 2012). doi:10.1109/tns.2011.2172629. 
Dose quantification in carbon ion therapy using in-beam PET

URL https://doi.org/10.1109/tns.2011.2172629

[61] D. W. Marquardt, An Algorithm for Least-Squares Estimation of Nonlinear Parameters, Journal of the Society for Industrial and Applied Mathematics 11 (2) (1963) 431-441 (Jun. 1963). doi:10.1137/0111030.

URL https://doi.org/10.1137/0111030

[62] A. Kraan, S. Muraro, G. Battistoni, N. Belcari, M. Bisogni, N. Camarlinghi, A. D. Guerra, A. Ferrari, R. Kopec, D. Krzempek, M. Morrocchi, P. Olko, P. Sala, K. Skowroǹska, G. Sportelli, A. Topi, V. Rosso, Analysis of in-beam PET time-profiles in proton therapy, Journal of Instrumentation 14 (02) (2019) C02001-C02001 (Feb. 2019). doi:10.1088/17480221/14/02/c02001.

URL https://doi.org/10.1088/1748-0221/14/02/c02001

[63] F. Attanasi, N. Belcari, S. Moehrs, V. Rosso, S. Vecchio, G. A. P. Cirrone, G. Cuttone, P. Lojacono, F. Romano, N. Lanconelli, A. D. Guerra, Characterization of an In-Beam PET Prototype for Proton Therapy With Different Target Compositions, IEEE Transactions on Nuclear Science 57 (3) (2010) 1563-1569 (Jun. 2010). doi:10.1109/tns.2010.2048124.

URL https://doi.org/10.1109/tns.2010.2048124

[64] S. Chu, L. Ekström, R. Firestone, The Lund/LBNL Nuclear Data Search, nuclide Search. 